\title{
Discussion of the Improved Methods for Analyzing a Cantilever Beam Carrying a Tip-Mass under Base Excitation
}

\author{
Wang Hongjin, ${ }^{1}$ Meng Qingfeng, ${ }^{1}$ and Feng Wuwei ${ }^{2}$ \\ ${ }^{1}$ Theory of Lubrication and Bearing Institute, Xian Jiaotong University, No. 28 Xianning West Road, Xian, Shaanxi 710049, China \\ ${ }^{2}$ School of Ship and Ocean Engineering, Zhejiang Ocean University, No. 18 Haiyuan Road, Zhoushan, Zhejiang 316000, China \\ Correspondence should be addressed to Wang Hongjin; whjin1984@126.com
}

Received 27 December 2012; Accepted 24 May 2013; Published 27 April 2014

Academic Editor: Reza Jazar

Copyright (c) 2014 Wang Hongjin et al. This is an open access article distributed under the Creative Commons Attribution License, which permits unrestricted use, distribution, and reproduction in any medium, provided the original work is properly cited.

\begin{abstract}
Two improved analytical methods of calculations for natural frequencies and mode shapes of a uniform cantilever beam carrying a tip-mass under base excitation are presented based on forced vibration theory and the method of separation of variables, respectively. The cantilever model is simplified in detail by replacing the tip-mass with an equivalent inertial force and inertial moment acting at the free end of the cantilever based on D'Alembert's principle. The concentrated equivalent inertial force and inertial moment are further represented as distributed loads using Dirac Delta Function. In this case, some typical natural frequencies and mode shapes of the cantilever model are calculated by the improved and unimproved analytical methods. The comparing results show that, after improvement, these two methods are in extremely good agreement with each other even the offset distance between the gravity center of the tip-mass and the attachment point is large. As further verification, the transient and steady displacement responses of the cantilever system under a sine base excitation are presented in which two improved methods are separately utilized. Finally, an experimental cantilever system is fabricated and the theoretical displacement responses are validated by the experimental measurements successfully.
\end{abstract}

\section{Introduction}

Laura et al. [1] successfully determined the first ten natural frequencies of a clamped-free beam with a finite mass at the free end using the standard method of separation of variables. Rama Bhat and Wagner [2] considered the frequencies of a uniform cantilever with an end mass using a power series expansion. [3] extended the research and presented the natural frequencies and mode shapes of a cantilever beam with a tip-mass and a base excitation by forced vibration theory. The consistencies of the analysis results derived by two different analytical methods separately from $[1,3]$ were discussed in $[4,5]$. Finally, Jacquot [6] concluded and proved that the results of the mode shapes of the cantilever beam carrying a tip mass were both correct $[1,3]$. Recently, Mousavi Lajimi and Heppler [7] provided a further discussion on [2] and made the method more complete. Soon Bhat [8] gave a response to the author of [7]. On the other hand, based on the model from [3], Esmailzadeh and Nakhaie-Jazar [9] derived the equation of vibration motion of a cantilever beam with a lumped mass while being excited harmonically at the base. Abramovich and Hamburger [10] further extended the research through the theory of Timoshenko beam. In recent, this model has been used for the application of vibration control [11] and energy harvesting [12]. However, the conclusions and evidences above can only prove the consistency of the methods of forced vibration and separation of variables for analyzing the cantilever beam when the gravity center of the tip-mass coincided with the point of the end attachment.

In this paper, we reinforced the method of separation of variables from [1] and developed the forced vibration method from [3]. Then, the natural frequencies and mode shapes of a cantilever with an offset end-mass under transverse excitation are derived using both of the improved analytical methods. In addition, the tip-mass is equated by an inertial force and inertial moment acting at the free end of the cantilever beam based on D'Alembert's principle and the equivalent process is detailed. The external loads including the concentrated inertia force, the concentrated inertia 


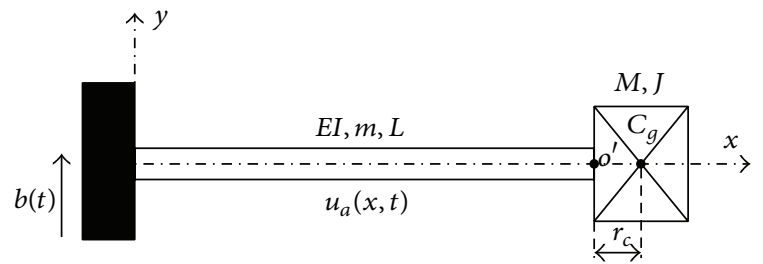

FIGURE 1: The model of cantilever beam with a tip-mass under base excitation.

moment, and the base excitation are represented as distributed loads using Dirac Delta Function. In this case, some typical natural frequencies and mode shapes are calculated by the developed and undeveloped methods. The comparing results show that the inconsistency between the mode shapes derived by the undeveloped method become larger with the increasing offset distance. However, after improvement, the mode shapes derived by two developed methods are in extremely good agreement with each other even the offset distance between the gravity center of the tip-mass and the attachment point is large. As further verification, the transient and steady displacement responses of the cantilever system under a sine base excitation are presented by modal analysis method in which the mode shapes derived by two improved analytical methods above are separately utilized. Finally, an experimental cantilever system is fabricated and the theoretical displacement responses are validated by the experimental measurements successfully.

\section{The Mathematical Model}

The uniform cantilever beam carrying a tip-mass at the free end under base excitation is shown schematically in Figure 1. It should be noticed that the gravity center of the tip-mass is collinear with the gravity center of beam but does not coincide with the point of the end attachment $o^{\prime}$. The mass and the moment of inertia of the tip-mass are both taken into account, but the rotary inertia of the beam is neglected.

2.1. The Simplified Model of the Cantilever Beam with a TipMass. According to D'Alembert's principle, the tip-mass at the free end of the cantilever can be equated by an inertial force and an inertial moment while vibrating. In addition, the tip-mass with larger elastic modulus is always chosen comparing to the elastic modulus of beam. So during vibration, the deformation of tip-mass can be ignored comparing to the beam. That implies that the tip-mass can be regarded as a rigid body while vibrating. In this case, considering the translational motion and rotational motion about a fixed axis of rigid body, the tip-mass can be equated by an inertial force and an inertial moment. The details about these two equivalent processing will be discussed as follows.

In the following analysis, we will limit our study about the tip-mass to planar kinetics because the rigid tip-mass can be considered to be symmetrical with respect to a fixed reference plane.
First, considering the translational effect, since the motion of the rigid body can be viewed within the reference plane, all the forces and couple moments acting on the body can then be projected into the plane. All particles inside the tip-mass may move with the same acceleration (see Figure 2(a)). The results of the simplification of this inertial force system to the point $o^{\prime}$ under translational motion give an inertial force and an inertial moment as follows:

$$
\begin{aligned}
\mathbf{F}_{g}^{(1)} & =\sum \mathbf{F}_{g i}^{(1)}=-\sum m_{i} \mathbf{a}_{i}=-\sum m_{i} \mathbf{a}_{c}=-M \mathbf{a}_{c}, \\
\mathbf{M}_{g}^{(1)} & =\sum \mathbf{r}_{i} \times \mathbf{F}_{g i}^{(1)}=-\sum \mathbf{r}_{i} m_{i} \times \mathbf{a}_{c}=-M \mathbf{r}_{c} \times \mathbf{a}_{c},
\end{aligned}
$$

where the vector $\mathbf{a}_{c}$ indicates the acceleration of the gravity center of the tip-mass, the parameter $M$ indicates the value of the tip-mass, and $\mathbf{r}_{c}$ presents the offset distance.

Next, in the condition of rotational motion about an axis perpendicular to the reference plane and passing through point $o^{\prime}$, all particles inside the rigid tip-mass may rotate with the same angular acceleration (see Figure 2(b)). Reducing the inertial rotating system to point $o^{\prime}$ gives the other inertial force and inertial moment as

$$
\begin{aligned}
\mathbf{F}_{g}^{(2)} & =\sum \mathbf{F}_{g i}^{(2)}=-\sum m_{i} \mathbf{a}_{i}^{\tau}=-\sum m_{i}\left(\boldsymbol{\xi} \times \mathbf{r}_{i}\right)=-M \boldsymbol{\xi} \times \mathbf{r}_{c}, \\
\mathbf{M}_{g}^{(2)} & =\sum \mathbf{r}_{i} \times \mathbf{F}_{i}^{(2)}=-\sum m_{i}\left(\mathbf{r}_{i} \times \boldsymbol{\xi} \times \mathbf{r}_{i}\right)=-\boldsymbol{\xi} \sum m_{i} r_{i}^{2} \\
& =-\boldsymbol{\xi}\left(J+M r_{c}^{2}\right),
\end{aligned}
$$

where $\xi$ and $J$ indicate the angular acceleration and the moment of inertia of the rigid tip-mass, respectively.

In this case, the total inertial force and inertial moment of the tip-mass can be written as the sum of the inertial equivalent forces and moments from translational and rotational motions. Assuming that the acceleration and angular acceleration of all particles inside of tip-mass are equal to the accelerations of the particle at point $o^{\prime}$, then the inertial equivalent force and moment of the rigid tip-mass would be written in scalar quantity as

$$
\begin{aligned}
F_{g} & =M \frac{\partial^{2} u_{a}(L, t)}{\partial t^{2}}+r_{c} M \frac{\partial^{2}}{\partial t^{2}}\left[\frac{\partial u_{a}(L, t)}{\partial x}\right], \\
M_{g} & =r_{c} M \frac{\partial^{2} u_{a}(L, t)}{\partial t^{2}}+\left(J+r_{c}^{2} M\right) \frac{\partial^{2}}{\partial t^{2}}\left[\frac{\partial u_{a}(L, t)}{\partial x}\right],
\end{aligned}
$$

where $u_{a}(L, t)$ indicates the absolute transverse displacement at the free end of the cantilever beam. The directions of the whole inertial equivalent force and moment can be found according to Newton's third law of motion as shown in Figure 3.

2.2. The Reinforced Method of Separation of Variables. In this section, the natural frequencies and mode shapes of the cantilever model (as shown in Figure 3) will be derived by the reinforced method of separation of variables. First, using Dirac Delta Function [13], the concentrated equivalent inertia 


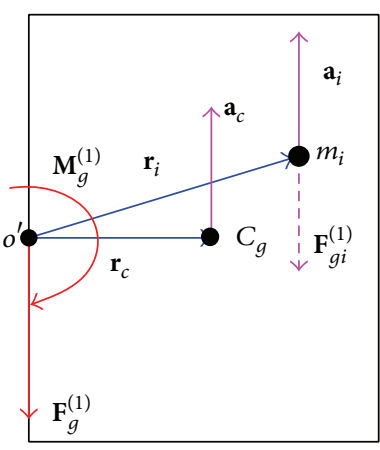

(a)

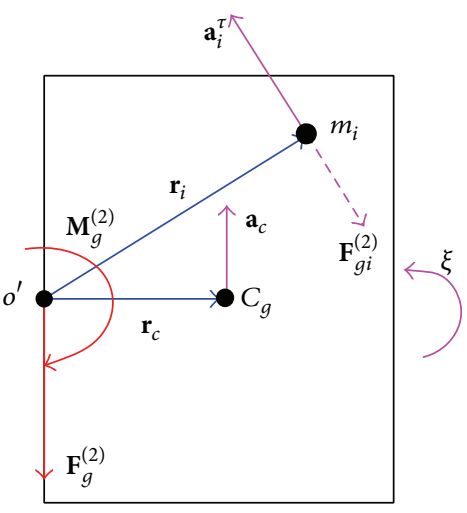

(b)

FIGURE 2: Simplification of the tip-mass to point $o^{\prime}$ under (a) translational motion and (b) rotational motion.

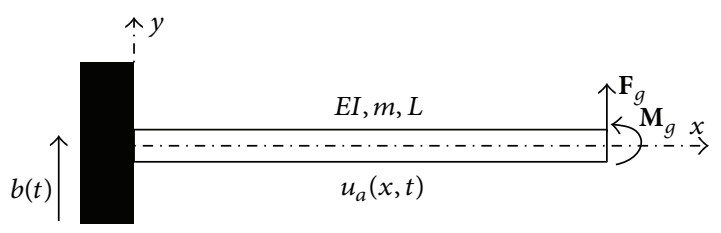

Figure 3: The simplified model of cantilever with tip-mass being equated by inertial force and moment.

force $F_{g}$ and inertia moment $M_{g}$ can be represented in the form of distributed loads as

$$
\begin{aligned}
f_{a}(x, t) & \\
= & F_{g} \delta(x-L) \\
= & {\left[M \frac{\partial^{2} u_{a}(x, t)}{\partial t^{2}}+r_{c} M \frac{\partial^{2}}{\partial t^{2}}\left(\frac{\partial u_{a}(x, t)}{\partial x}\right)\right] \delta(x-L), } \\
M_{a}(x, t) & \\
= & M_{g} \delta(x-L) \\
= & {\left[r_{c} M \frac{\partial^{2} u_{a}(x, t)}{\partial t^{2}}+\left(J+r_{c}^{2} M\right) \frac{\partial^{2}}{\partial t^{2}}\left(\frac{\partial u_{a}(x, t)}{\partial x}\right)\right] } \\
& \quad \times \frac{d \delta(x-L)}{d x} .
\end{aligned}
$$

Therefore, employing variational method, the dynamic motion equation of the simplified model of the cantilever beam with a tip-mass under base transverse excitation is obtained as

$$
E I \frac{\partial^{4} u_{a}(x, t)}{\partial x}+m \frac{\partial^{2} u_{a}(x, t)}{\partial t}=f_{a}(x, t)-M_{a}(x, t),
$$

where $E I$ and $m$ denote the flexural rigidity and the mass per unit length of the beam, respectively.
The absolute displacement can be considered as the sum of the relative displacement and the base displacement excitation:

$$
u_{a}(x, t)=u_{\text {rel }}(x, t)+b(t) .
$$

Substituting (6) into (5) gives the new form of the dynamic motion equation as

$$
\begin{aligned}
E I \frac{\partial^{4} u_{\text {rel }}(x, t)}{\partial x}+m \frac{\partial^{2} u_{\text {rel }}(x, t)}{\partial t} & \\
=[ & \left.M \frac{\partial^{2} u_{\text {rel }}(x, t)}{\partial t^{2}}+r_{c} M \frac{\partial^{2}}{\partial t^{2}}\left(\frac{\partial u_{\text {rel }}(x, t)}{\partial x}\right)\right] \delta(x-L) \\
& +\left[r_{c} M \frac{\partial^{2} u_{\text {rel }}(x, t)}{\partial t^{2}}+\left(J+r_{c}^{2} M\right) \frac{\partial^{2}}{\partial t^{2}}\left(\frac{\partial u_{\text {rel }}(x, t)}{\partial x}\right)\right] \\
& \times \frac{d \delta(x-L)}{d x} \\
& +\left[M \delta(x-L)+r_{c} M \frac{d \delta(x-L)}{d x}-m\right] \frac{d^{2} b(t)}{d t^{2}} .
\end{aligned}
$$

In this case, the simplified model of the cantilever beam under base excitation can be considered as a new analytical model with the dynamic differential equation of (8) and the boundary conditions of (9) through (12):

$$
\begin{aligned}
E I & \frac{\partial^{4} u_{a}(x, t)}{\partial x}+m \frac{\partial^{2} u_{a}(x, t)}{\partial t} \\
& =\left[M \delta(x-L)+r_{c} M \frac{d \delta(x-L)}{d x}-m\right] \frac{d^{2} b(t)}{d t^{2}}
\end{aligned}
$$

at $x=0$,

$$
\begin{gathered}
u_{a}(x, t)=0, \\
\frac{d}{d x} u_{a}(x, t)=0
\end{gathered}
$$




$$
\begin{aligned}
& \text { at } x=L, \\
& E I \frac{d^{3}}{d x^{3}} u_{a}(x, t)=\left[M \frac{\partial^{2} u_{a}(x, t)}{\partial t^{2}}+r_{c} M \frac{\partial^{2}}{\partial t^{2}}\left(\frac{\partial u_{a}(x, t)}{\partial x}\right)\right] \\
& E I \frac{d^{2}}{d x^{2}} u_{a}(x, t) \\
& =-\left[r_{c} M \frac{\partial^{2} u_{a}(x, t)}{\partial t^{2}}+\left(J+r_{c}^{2} M\right) \frac{\partial^{2}}{\partial t^{2}}\left(\frac{\partial u_{a}(x, t)}{\partial x}\right)\right] .
\end{aligned}
$$

The solutions of (8) subjected to four boundary conditions and two initial conditions can be obtained conveniently by the traditional method of separation of variables. The method regards the response as a superposition of the system eigen-functions multiplied by corresponding time dependent generalized coordinates. Of course, this necessitates first obtaining the solution of the system eigen-value problem. Turning attentions to the corresponding eigen-value problem and considering the free vibration characterized by setting $b(t)=0$, the solution of (8) becomes separable in space and time. Letting

$$
u_{a}(x, t)=U(x) T(t)
$$

and substituting (13) into the free vibration form of (8), two dependent equations are obtained using the separation of variables method as

$$
\begin{gathered}
\frac{d}{d t^{2}} T(t)+\omega^{2} T(t)=0, \\
\frac{d^{4}}{d x^{4}} U(x)-\beta^{4} U(x)=0,
\end{gathered}
$$

where $\omega^{2}$ indicates the angular frequency and $\beta^{4}=$ $m \omega^{2} /(E I)$. In this case, the four boundary conditions will be changed as

$$
\begin{gathered}
\text { at } x=0, \\
U(x)=0 \\
\frac{d}{d x} U(x)=0 \\
\text { at } x=L, \\
E I \frac{d^{3}}{d x^{3}} U(x)=-\left[\omega^{2} M U(x)+r_{c} M \omega^{2}\left(\frac{d}{d x} U(x)\right)\right] \\
E I \frac{d^{2}}{d x^{2}} U(x)=r_{c} M \omega^{2} U(x)+\left(J+r_{c}^{2} M\right) \omega^{2}\left(\frac{d}{d x} U(x)\right)
\end{gathered}
$$

The general solution of (15) can be easily derived to be

$$
\begin{aligned}
U(x)= & C_{1} \cos (\beta x)+C_{2} \sin (\beta x)+C_{3} \cosh (\beta x) \\
& +C_{4} \sinh (\beta x)
\end{aligned}
$$

where $C_{n}(n=1,2,3,4)$ are constants of integration. In order to evaluate three of these constants in terms of the fourth and derive the characteristic equation, the boundary conditions (16) to (19) must be used. Indeed, substituting (20) into (16) through (19) gives the characteristic equation and the mode shapes as

$$
\begin{gathered}
1+c c h-\phi u(s c h-c s h) \\
-R_{1}[s c h+c s h-\phi u(1-c c h)] \\
-2 R_{2} s s h-R_{2}^{2}(1-c c h)=0, \\
U(x)=C_{2}\{\sin (\beta x)-\operatorname{sh}(\beta x)-\lambda[\cos (\beta x)-\operatorname{ch}(\beta x)]\},
\end{gathered}
$$

where $\lambda=A_{12} / A_{11}$ and the constant $C_{2}$ is an arbitrary value and in which

$$
\begin{aligned}
& A_{11}=-\left(1+R_{2}\right) c+\left(R_{2}-1\right) c h+R_{1}(s+s h), A_{12}= \\
& R_{1}(c h-c)-\left(1+R_{2}\right) s+\left(R_{2}-1\right) s h, \\
& u=\beta L, s=\sin (u), c=\cos (u), s h=\sinh (u), c h= \\
& \cosh (u), \\
& \phi=M /(m L), R_{1}=\phi u^{3}\left[(K / L)^{2}+\left(r_{c} / L\right)^{2}\right], R_{2}= \\
& \phi u^{2}\left(r_{c} / L\right), K=\sqrt{J / M} .
\end{aligned}
$$

2.3. The Developed Method of Forced Vibration. If we treat the base displacement excitation as a kind of boundary condition, then the differential equation of motion and four boundary conditions of the cantilever system can be written as

$$
E I \frac{\partial^{4} u_{a}(x, t)}{\partial x}+m \frac{\partial^{2} u_{a}(x, t)}{\partial t}=0
$$

at $x=0$,

$$
\begin{aligned}
& u_{a}(0, t)=b(t), \\
& \frac{d}{d x} u_{a}(0, t)=0,
\end{aligned}
$$

at $x=L$,

$$
\begin{aligned}
& E I \frac{d^{3}}{d x^{3}} u_{a}(x, t) \\
& \quad=\left[M \frac{\partial^{2} u_{a}(x, t)}{\partial t^{2}}+r_{c} M \frac{\partial^{2}}{\partial t^{2}}\left(\frac{\partial u_{a}(x, t)}{\partial x}\right)\right] \\
& E I \frac{d^{2}}{d x^{2}} u_{a}(x, t) \\
& \quad=-\left[r_{c} M \frac{\partial^{2} u_{a}(x, t)}{\partial t^{2}}+\left(J+r_{c}^{2} M\right) \frac{\partial^{2}}{\partial t^{2}}\left(\frac{\partial u_{a}(x, t)}{\partial x}\right)\right]
\end{aligned}
$$


Under the simple harmonic excitation $e^{j \omega t}$, the steady state solution of (23) can be regarded with the form as

$$
\begin{aligned}
u_{a}(x, t) & =U_{a}(x, \omega) e^{j \omega t-\varphi} \\
= & {\left[B_{1} \cos (\beta x)+B_{2} \sin (\beta x)\right] e^{j \omega t-\varphi} } \\
& \left.+B_{3} \cosh (\beta x)+B_{4} \sinh (\beta x)\right],
\end{aligned}
$$

where $B_{n}(n=1,2,3,4)$ are arbitrary constants, the parameter $\beta$ is defined by $\beta^{4}=m \omega^{2} /(E I), j$ is the imaginary unit and $\varphi$ is the lagging phase.

We recalculated the natural frequencies and the mode shapes directly by substituting (28) into four boundary conditions (24) through (27) and obtained the characteristic equation and displacement response which are different from [3]:

$$
\begin{aligned}
D= & 1+c c h-\phi u(s c h-c s h) \\
& -R_{1}[s c h+c s h-\phi u(1-c c h)] \\
& -2 R_{2} s s h-R_{2}^{2}(1-c c h), \\
U(x)= & \left(\frac{B}{2 D}\right)\left\{F_{1 u}^{\prime} \cos (\beta x)+F_{2 u}^{\prime} \cosh (\beta x)\right. \\
& \left.+F_{3 u}^{\prime}[\sin (\beta x)-\sinh (\beta x)]\right\},
\end{aligned}
$$

where $B$ is defined by $b(t)=B e^{j \omega t}$, and in which

$$
\begin{aligned}
& F_{1 u}^{\prime}=1+c c h-s s h-2 \varphi u s c h-2 R_{1} c s h+\left(R_{1} \varphi u-R_{2}^{2}\right)(1- \\
& c c h+s s h)-2 R_{2}(c c h+s s h)=F_{1 u}-2 R_{2}(c c h+s h), \\
& F_{2 u}^{\prime}=1+c c h+s s h+2 \varphi u c s h-2 R_{1} s c h+\left(R_{1} \varphi u-R_{2}^{2}\right)(1- \\
& c c h-s s h)+2 R_{2}(c c h-s s h)=F_{2 u}+2 R_{2}(c c h-s s h), \\
& F_{3 u}^{\prime}=(s c h+c s h)\left(1-R_{1} \varphi u+R_{2}^{2}\right)+2\left[\varphi u c c h-R_{1} s s h-\right. \\
& \left.R_{2}(s c h-c s h)\right]=F_{3 u} .
\end{aligned}
$$

It is obvious that the characteristic equation derived by the reinforced method of separation of variables is equal to the equation obtained by the developed method of forced vibration.

The mode shapes cannot be obtained directly because the boundary conditions contain the parameter $\omega^{2}$. However, this problem can be successfully solved by Dirac Delta Function [13]. Thus, the mode shapes may be obtained by the SturmLiouville theory [14] and expressed as

$$
\begin{array}{r}
U_{i}(x)=\left(\frac{1}{2}\right)\left\{F_{1 u_{i}}^{\prime} F \cos \left(\beta_{i} x\right)+F_{2 u_{i}}^{\prime} \cosh \left(\beta_{i} x\right)\right. \\
\left.+F_{3 u_{i}}^{\prime}\left[\sin \left(\beta_{i} x\right)-\sinh \left(\beta_{i} x\right)\right]\right\} .
\end{array}
$$

\section{Computed Results and Comparisons}

In this section, three types of the natural mode shapes of the cantilever beam with a tip-mass were computed and compared. The first one is called the undeveloped mode shapes which is directly obtained from [3] by the expression

$$
\begin{array}{r}
U_{i}^{(1)}(x)=\left(\frac{1}{2}\right)\left\{F_{1 i c} \cos \left(\frac{u_{i} x}{L}\right)+F_{2 i c} \cosh \left(\frac{u_{i} x}{L}\right)\right. \\
\left.+F_{3 i c}\left[\sin \left(\frac{u_{i} x}{L}\right)-\sinh \left(\frac{u_{i} x}{L}\right)\right]\right\},
\end{array}
$$

where $F_{1 i c}=F_{1 i} / \operatorname{ch}\left(u_{i}\right), F_{2 i c}=F_{2 i} / \operatorname{ch}\left(u_{i}\right)$, and $F_{3 i c}=$ $F_{3 i} / \operatorname{ch}\left(u_{i}\right)$, in which $F_{1 i}, F_{2 i}$, and $F_{3 i}$ are the functions $F_{1 u}, F_{2 u}$, and $F_{3 u}$ with the variable $u$ replaced by $u_{i}$, respectively.

The second one is based on the expression of the developed mode shapes derived in this paper. Let (30) be multiplied by $2 / F_{3 i}^{\prime}$ to give the second mode shapes as

$$
\begin{aligned}
U_{i}^{(2)}(x)= & \sin \left(\frac{u_{i} x}{L}\right)-\sinh \left(\frac{u_{i} x}{L}\right)+\left(\frac{F_{1 i}^{\prime}}{F_{3 i}^{\prime}}\right) \cos \left(\frac{u_{i} x}{L}\right) \\
& +\left(\frac{F_{2 i}^{\prime}}{F_{3 i}^{\prime}}\right) \cosh \left(\frac{u_{i} x}{L}\right) .
\end{aligned}
$$

At last, the arbitrary constant $C_{2}$ in (22) was chosen as constant 1 to give the third type of the natural mode shapes as

$$
\begin{aligned}
U_{i}^{(3)}(x)= & \sin \left(\frac{u_{i} x}{L}\right)-\operatorname{sh}\left(\frac{u_{i} x}{L}\right) \\
& -\lambda\left[\cos \left(\frac{u_{i} x}{L}\right)-\operatorname{ch}\left(\frac{u_{i} x}{L}\right)\right] .
\end{aligned}
$$

Before the mode shapes are determined, the roots of the characteristic equation must be calculated firstly. The characteristic equation (21) or $D=0$ can be solved by two steps. First, approximate roots were pointed out by applying graphing method. Then, the approximate roots were iterated by the bisection method to give the exact characteristic roots with given error. All these processes were finished using MATLAB software.

Because the condition that the gravity center of the tipmass coincided with the point of the end attachment has been verified in [6], we focus on the condition when the offset distance is not zero. Table 1 shows the first five roots of the characteristic equations from five samples with different values of $\phi, k / L$, and $d / L$. It can be clearly noticed that the larger value of $d / L$ resulted in the less value of the first characteristic root but the larger values of the other four characteristic roots when $\phi$ and $k / L$ were set as constants.

Once the roots of the characteristic equation have been obtained, three types of the first five mode shapes of the cantilever structure from the five given samples can be calculated. Figures 4, 5, 6, 7, and 8 show the first five mode shapes of the cantilever with various combinations of $\phi, k / L$, and $d / L$. 
TABLE 1: Typical roots of characteristic equation with variables $\phi, k / L$, and $d / L$.

\begin{tabular}{lcccccccc}
\hline Sample & $\phi$ & $K / L$ & $d / L$ & $u_{1}$ & \multicolumn{3}{c}{ Roots of characteristic equation } & $u_{2}$ \\
\hline 1 & 4 & 4 & 0 & 0.35211 & 1.29234 & 4.77818 & 7.88401 & 11.01780 \\
2 & 4 & 4 & 0.5 & 0.34814 & 1.30637 & 4.77952 & 7.88472 & 11.01827 \\
3 & 4 & 4 & 1 & 0.34200 & 1.32825 & 4.78225 & 7.88634 & 11.01940 \\
4 & 4 & 4 & 2 & 0.32544 & 1.38983 & 4.79177 & 7.89233 & 11.02368 \\
5 & 4 & 4 & 4 & 0.28806 & 1.54543 & 4.82543 & 7.91455 & 11.03990 \\
\hline
\end{tabular}
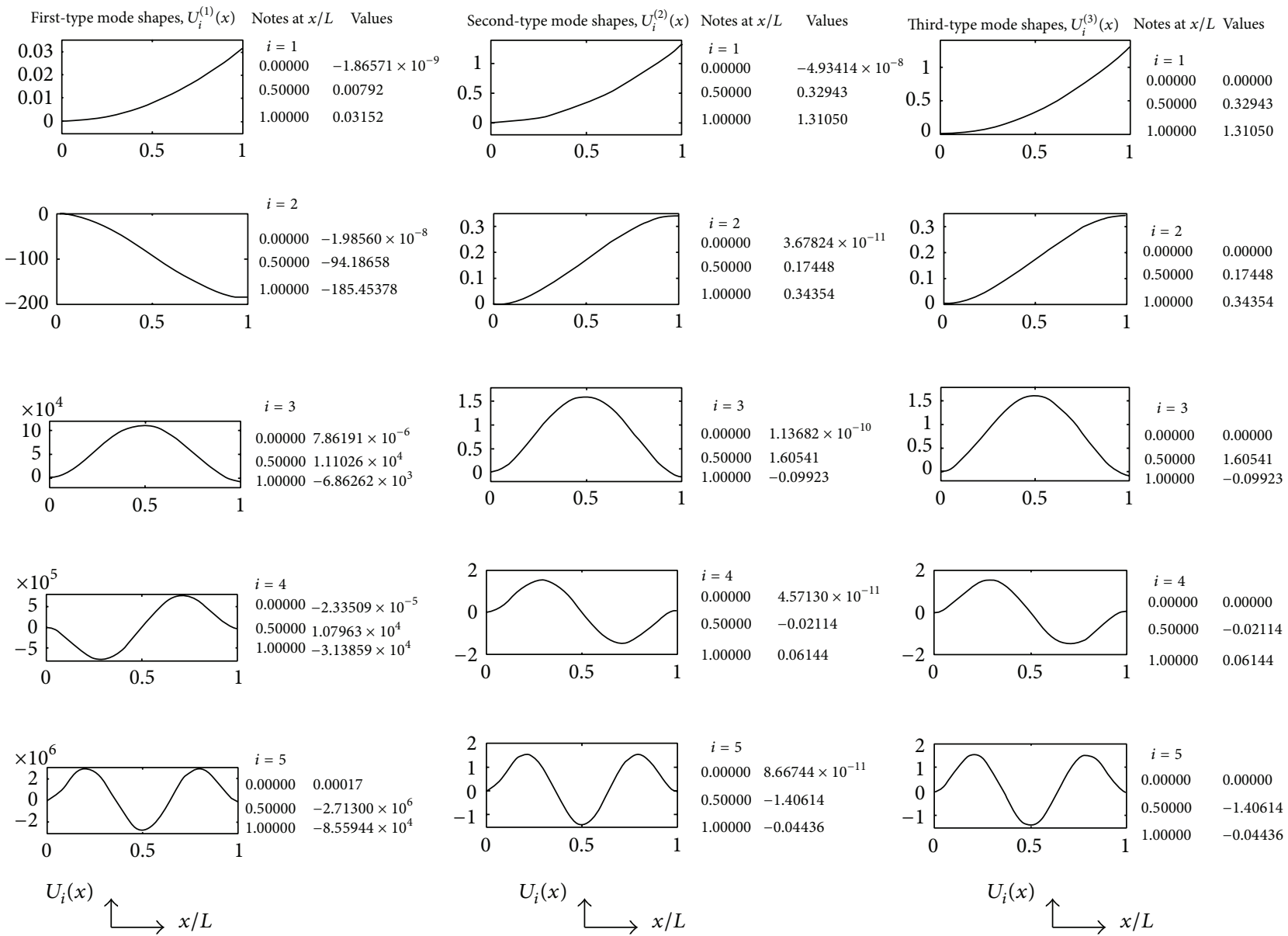

Figure 4: Three types of the first five mode shapes with the combinations of $\phi=4, k / L=4$, and $d / L=0$.

It is obvious that when $d / L \neq 0$ the first type of nature mode shapes $U_{i}^{(1)}(x)$ derived by the undeveloped method turned far away from the third-type mode shapes $U_{i}^{(3)}(x)$ derived by the reinforced method of separation of variables. In addition, the larger value of $d / L$ resulted in the more inconsistent mode shapes. However, the second type of mode shapes $U_{i}^{(2)}(x)$ obtained by the developed method of forced vibration seemed in good agreement with the third ones.

Furthermore, the amplitude of the first mode shape decreased, but the other four mode shapes increased with the increasing value of the $d / L$ when $k / L$ and $\phi$ were fixed to be constants. The effect from the varying value of $d / L$ for the lower mode shapes was always stronger than for the higher.

\section{Experimental Verification}

In order to experimentally explain and compare two types of natural mode shapes derived by two improved methods above, the transient and steady responses of the cantilever system under an input base excitation should be calculated first. This whole calculation can be finished by method of modal analysis. But above all, the orthogonality property of 

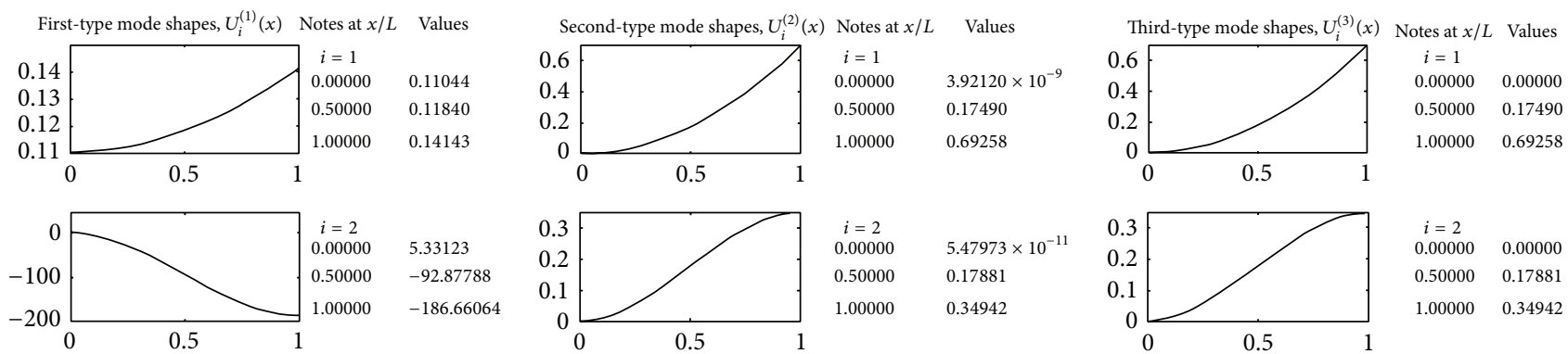

$i=2$

$0.00000 \quad 0.00000$

$0.50000 \quad 0.17881$

$1.00000 \quad 0.34942$
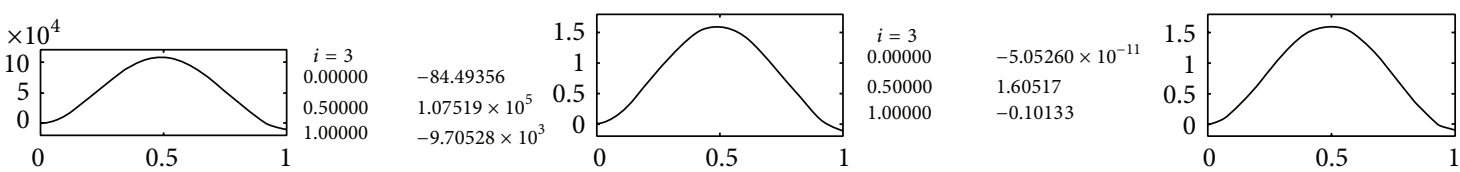

$i=3$

0.00000

$0.50000 \quad 1.60517$

$1.00000-0.10133$
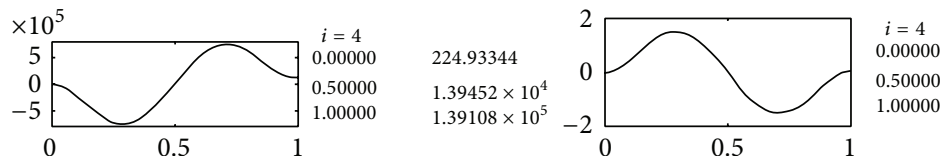

$-7.76048 \times 10^{-1}$

$-0.02162$

0.06261

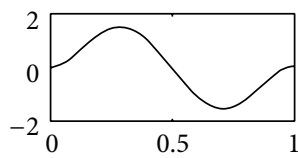

$i=4$
0.00000 $\quad 0.00000$

$0.50000 \quad-0.02162$

$1.00000 \quad 0.06261$
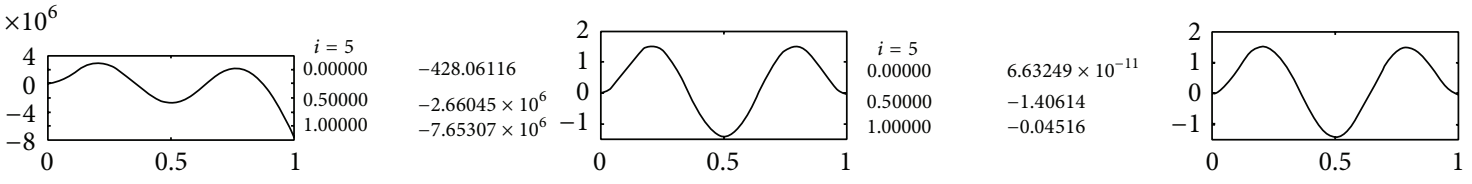

$i=5$

$0.00000-0.00000$

$6.63249 \times 10^{-11}$

$-1.40614$

$-0.04516$

$\begin{array}{ll}0.50000 & -1.40614 \\ 1.00000 & -0.04516\end{array}$

$U_{i}(x)$

$U_{i}(x)$

个 $x / L$

$U_{i}(x)$

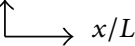

FIGURE 5: Three types of the first five mode shapes with the combinations of $\phi=4, k / L=4$, and $d / L=0.5$.
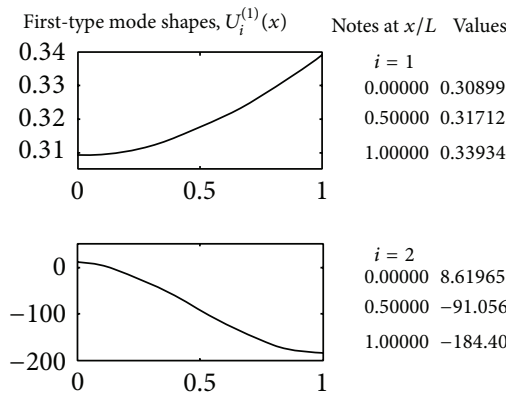

$i=2$

0.000008 .61965

$0.50000-91.05604$

$1.00000-184.40224$
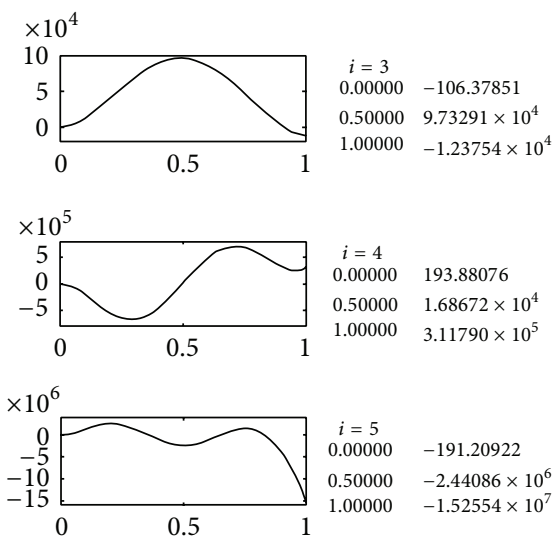

$U_{i}(x)$

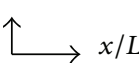

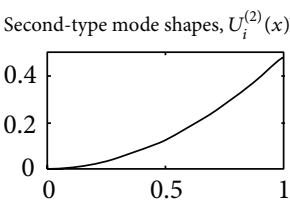

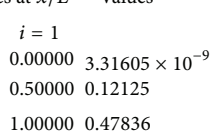

0.500000 .12125

1.000000 .47836

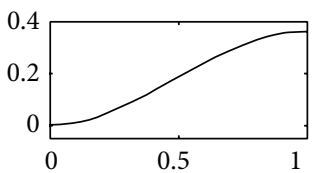

$i=2$

$0.00000-5.50109 \times 10^{-1}$

0.500000 .18658

$\begin{array}{lll}1.00000 & 0.36219\end{array}$

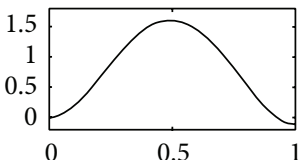

$i=3$
0.00000

$\begin{array}{ll}0.50000 & 1.60459\end{array}$

$\begin{array}{ll}1.00000 & -0.10631\end{array}$

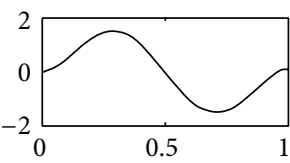

$i=4$

$0.00000 \quad 1.09777 \times 10^{-10}$

$0.50000-0.02275$

$\begin{array}{ll}1.00000 & 0.06562\end{array}$

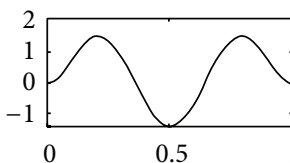

$i=5$

$0.00000 \quad 8.36395 \times 10^{-11}$

$\begin{array}{ll}0.50000 & -1.40614 \\ 1.00000 & -0.04731\end{array}$
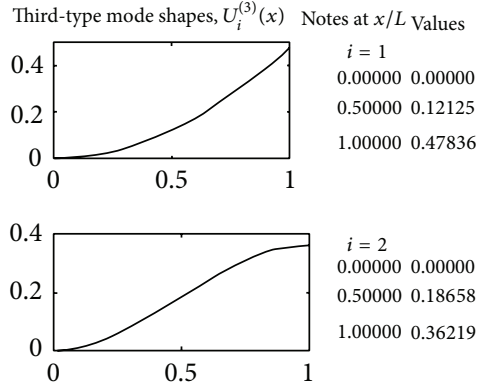

0.000000 .00000 0.500000 .18658

1.000000 .36219

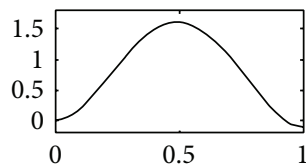

$i=3$

$0.00000 \quad 0.00000$

$0.50000 \quad 1.60459$ $\begin{array}{ll}1.00000 & -0.10631\end{array}$

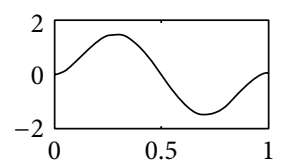

$i=4$

0.00000

$\begin{array}{ll}0.50000 & -0.02275\end{array}$

$\begin{array}{ll}1.00000 & 0.06562\end{array}$

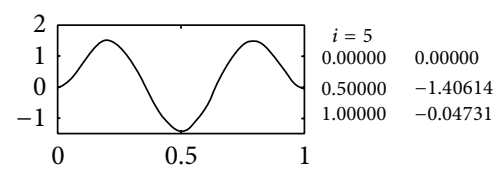

$U_{i}(x)$

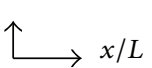

FIGURE 6: Three types of the first five mode shapes with the combinations of $\phi=4, k / L=4$, and $d / L=1$. 
First-type mode shapes, $U_{i}^{(1)}(x) \quad$ Notes at $x / L$ Values

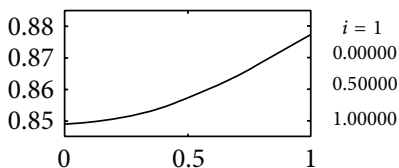

0.84906 0.85736

0.87731

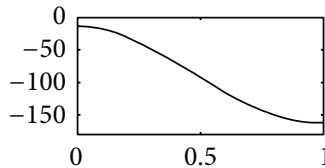

$i=2$

$0.00000-13.08377$

$0.50000-93.08045$

$1.00000-160.72843$

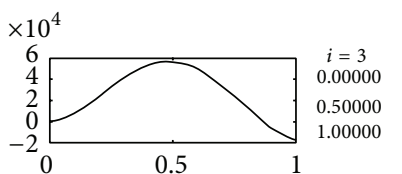

634.90847

$5.65642 \times 10^{4}$

$-1.72997 \times 10^{4}$
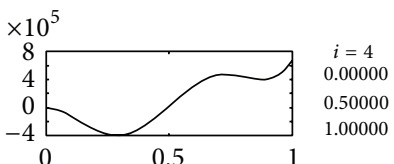

$-3297.62092$ $2.30021 \times 10^{4}$ $6.67852 \times 10^{5}$

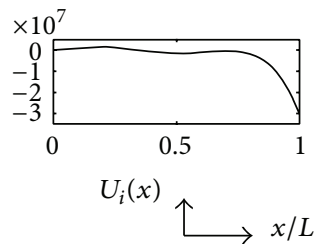

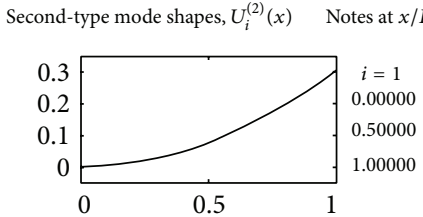

Values
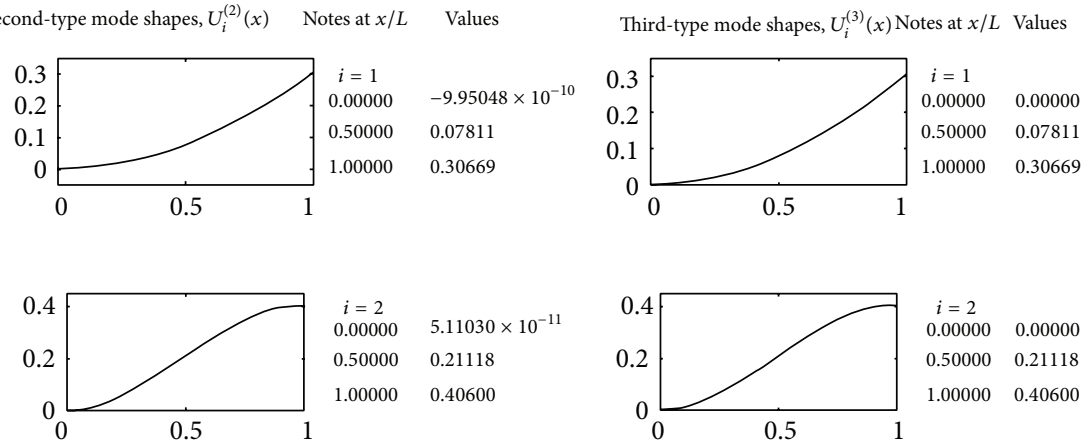

$i=2$ $0.00000 \quad 0.00000$ $\begin{array}{ll}0.50000 & 0.21118\end{array}$ $1.00000 \quad 0.40600$
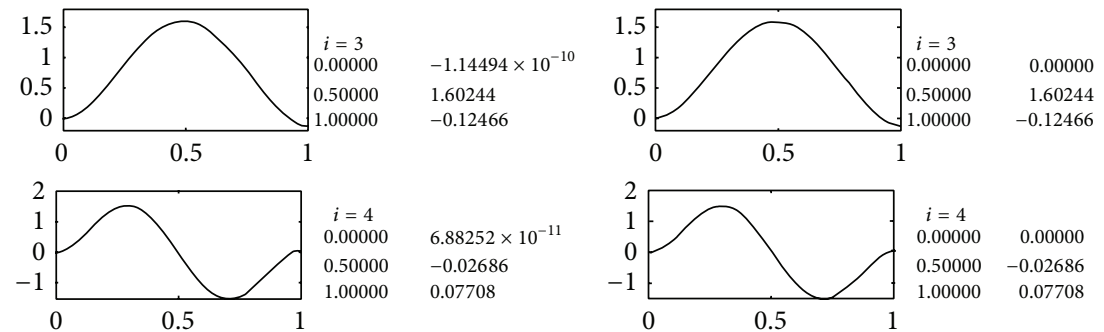

$6.88252 \times 10^{-11}$ $-0.02686$ 0.07708
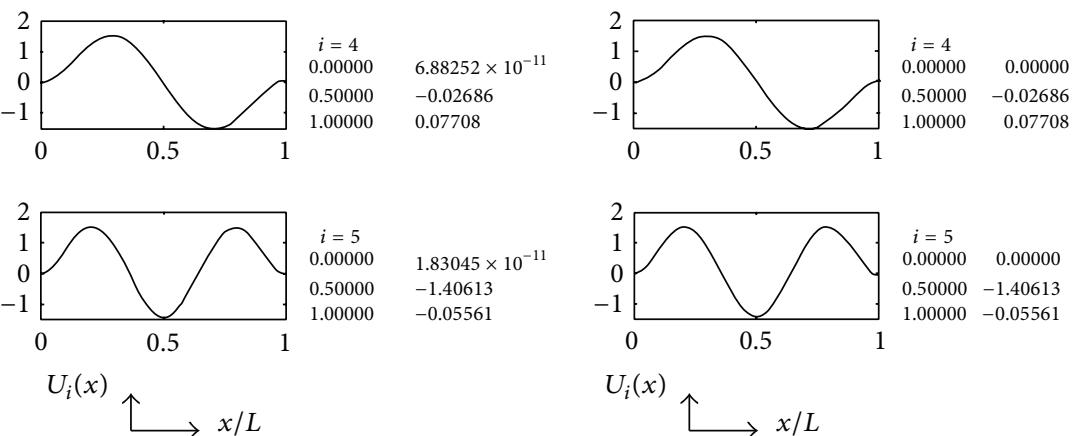

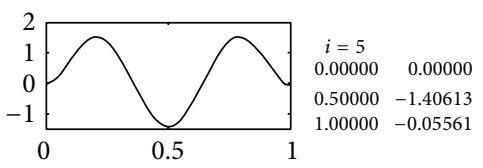

$U_{i}(x)$ 个 $x / L$

FIGURE 7: Three types of the first five mode shapes with the combinations of $\phi=4, k / L=4$, and $d / L=2$.

the natural mode shapes should be derived. In fact, using Betti theorem [15], the orthogonality properties with respect to mass and stiffness can be obtained, respectively, as follows:

$$
\begin{aligned}
\int_{0}^{L} m U_{r}^{(k)}(x) U_{i}^{(k)}(x) d x+M U_{r}^{(k)}(L) U_{i}^{(k)}(L) \\
+r_{c} M U_{i}^{(k)}(L) \frac{d U_{r}^{(k)}(L)}{d x}+r_{c} M U_{r}^{(k)}(L) \frac{d U_{i}^{(k)}(L)}{d x} \\
+\left(J+r_{c}^{2} M\right) \frac{d U_{i}^{(k)}(L)}{d x} \frac{d U_{r}^{(k)}(L)}{d x}=\left(\delta_{r i}^{(k)}\right)^{2}, \\
(k=1,2 ; r, i=1,2, \ldots),
\end{aligned}
$$

$$
\begin{array}{r}
\int_{0}^{L} E I \frac{d^{2} U_{i}^{(k)}(x)}{d x^{2}} \frac{d^{2} U_{r}^{(k)}(x)}{d x^{2}} d x=\left(\omega_{r} \delta_{r i}^{(k)}\right)^{2}, \\
(k=1,2 ; r, i=1,2, \ldots) .
\end{array}
$$

In this case, the normalized mode shapes can be defined as

$$
U_{r N}^{(k)}(x)=\frac{U_{r}^{(k)}(x)}{\delta_{r r}^{(k)}}
$$

where

$$
\begin{array}{rl}
\left(\delta_{r r}^{(k)}\right)^{2}=\int_{0}^{L} & m\left[U_{r}^{(k)}(x)\right]^{2} d x+M\left[U_{r}^{(k)}(L)\right]^{2} \\
& +2 r_{c} M U_{r}^{(k)}(L)\left[\frac{d}{d x} U_{r}^{(k)}(L)\right] \\
& +\left(J+M r_{c}^{2}\right)\left[\frac{d}{d x} U_{r}^{(k)}(L)\right]^{2}
\end{array}
$$

4.1. Theoretical Responses of the Cantilever System with a Base Sine Excitation. According to the method of modal analysis, the solution of (8) has the form of

$$
u_{a}(x, t)=\sum_{r=1}^{\infty} U_{r N}^{(k)}(x) q_{r}(t) .
$$

So, inserting (38) into (8) gives

$$
\begin{aligned}
E I \sum_{r=1}^{\infty} & \frac{d^{4} U_{r N}^{(k)}(x)}{d x^{4}} q_{r}(t)+m \sum_{r=1}^{\infty} U_{r N}^{(k)}(x) \frac{d^{2} q_{r}(t)}{d t^{2}} \\
& =\left[M \delta(x-L)+r_{c} M \frac{d \delta(x-L)}{d x}-m\right] \frac{d^{2} b(t)}{d t^{2}} .
\end{aligned}
$$

Considering the orthogonality properties of equations (34) and (35), let (39) be multiplied by $U_{i N}^{(k)}(x)$ and integrated over 

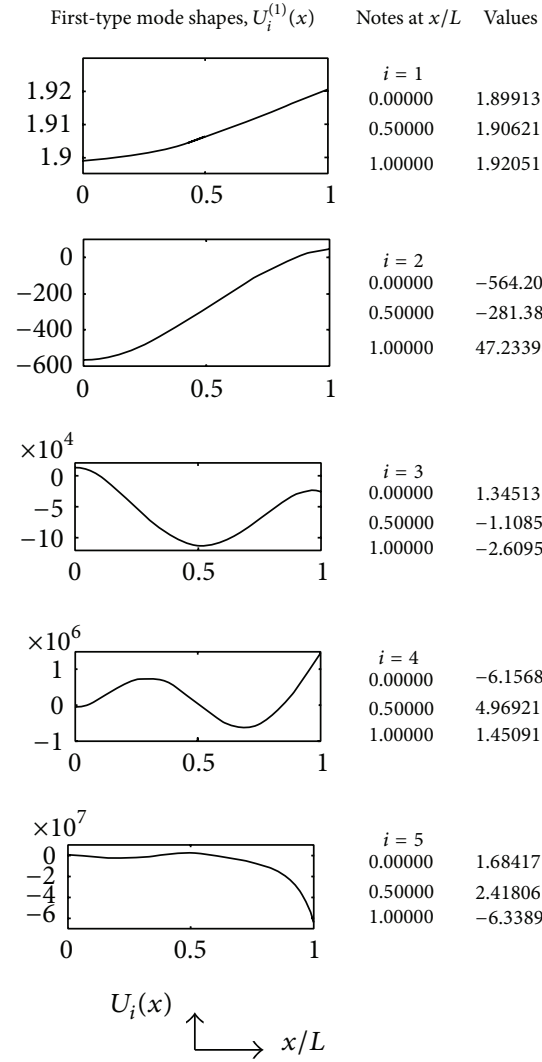

$i=4$

0.00000
0.50000

1.00000

$i=5$

$i=5$
0.00000

0.50000

1.00000
Second-type mode shapes, $U_{i}^{(2)}(x) \quad$ Notes at $x / L \quad$ Values

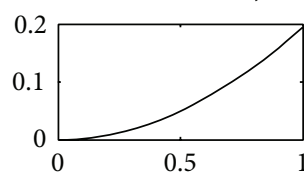

$i=1$

$0.00000 \quad 1.37503 \times 10^{-9}$

$0.50000 \quad 0.04964$

$1.00000 \quad 0.19458$

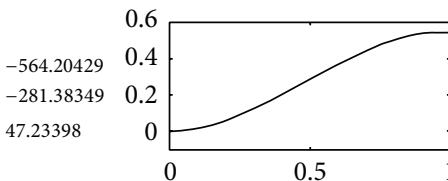

$\begin{array}{cl}i=2 & \\ 0.00000 & -2.38241 \times 10^{-1} \\ 0.50000 & 0.28530 \\ 1.00000 & 0.54402\end{array}$

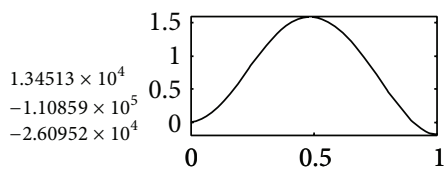

$i=3$
0.00000
0.50000
1.00000
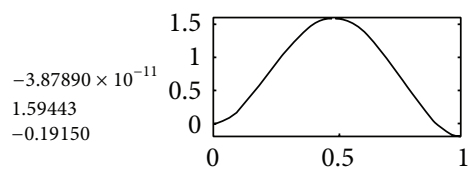

$i=3$

$0.00000 \quad 0.00000$

$0.50000 \quad 1.59443$

$\begin{array}{ll}1.00000 & -0.19150\end{array}$

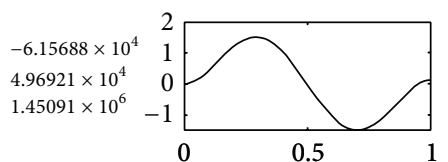
$i=4$
$0.00000-2.83947 \times 10^{-11}$
$0.50000-0.04212$
$\begin{array}{ll}1.00000 & 0.12050\end{array}$

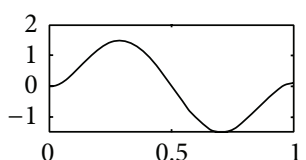

$i=4$

$0.00000 \quad 0.00000$

$0.50000 \quad-0.04212$

$1.00000 \quad 0.12050$

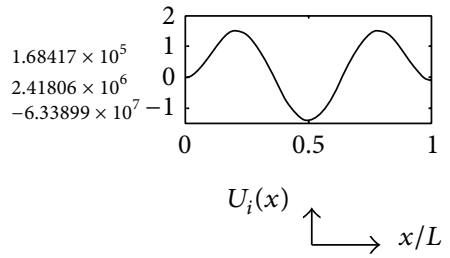
$\begin{array}{cl}i=5 & \\ 0.00000 & 7.71494 \times 10^{-11} \\ 0.50000 & -1.40605 \\ 1.00000 & -0.08755\end{array}$

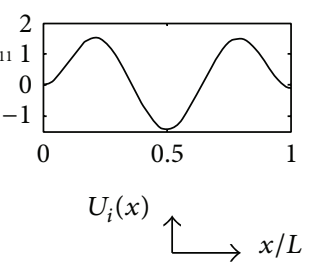

$i=5$

0.00000

$0.50000-1.40605$ $1.00000-0.08755$

FIGURE 8: Three types of the first five mode shapes with the combinations of $\phi=4, k / L=4$, and $d / L=4$.

the domain $0<x<L$ to give a set of independent ordinary differential equations as

$$
\frac{d^{2} q_{i}(t)}{d t^{2}}+\omega_{i}^{2} q_{i}(t)=f_{i}^{(k)}(t)
$$

Considering the properties of Dirac Delta Function

$$
\begin{gathered}
\int_{0}^{L} U_{i N}^{(k)}(x) \delta(x-L) d x=U_{i N}^{(k)}(L), \\
\int_{0}^{L} U_{i N}^{(k)}(x) \frac{d \delta(x-L)}{d x} d x=-\frac{d U_{i N}^{(k)}(L)}{d x},
\end{gathered}
$$

the generalized forces associated with the generalized coordinates $q_{i}(t)$ can be obtained as

$$
\begin{aligned}
& f_{i}^{(k)}(t) \\
& =\left[M U_{i N}^{(k)}(L)-r_{c} M \frac{d U_{i N}^{(k)}(L)}{d x}-m \int_{0}^{L} U_{i N}^{(k)}(x) d x\right] \frac{d^{2} b(t)}{d t^{2}} .
\end{aligned}
$$

Furthermore, considering the mechanical damping as Rayleigh damping and ignoring the part of mass damping, the damping coefficient $\zeta$ of the bimorph beam can be determined by the modal damping ratio which can be obtained by experiment [16]. The independent ordinary differential equations with damping then can be written as

$$
\begin{aligned}
& \frac{d^{2} q_{i}(t)}{d t^{2}}+2 \omega_{i} \zeta \frac{d q_{i}(t)}{d t}+\omega_{i}^{2} q_{i}(t) \\
& =\left[M U_{i N}^{(k)}(L)-r_{c} M \frac{d U_{i N}^{(k)}(L)}{d x}-m \int_{0}^{L} U_{i N}^{(k)}(x) d x\right] \frac{d^{2} b(t)}{d t^{2}} .
\end{aligned}
$$

Now, (43) can be solved using Laplace transform. Applying a sine excitation $b(t)=B \sin (\omega t)$ to the base of cantilever structure, under zero initial conditions, the transfer function of the independent differential equations for the cantilever beam with tip-mass under base displacement excitation can be written as

$G_{i}(s)$

$$
=\frac{Q_{i}(s)}{B_{i}(s)}
$$

$$
=\frac{\left(-\omega^{2}\right)\left[M U_{i N}^{(k)}(L)-r_{c} M\left(d U_{i N}^{(k)}(L) / d x\right)-m \int_{0}^{L} U_{i N}^{(k)}(x) d x\right]}{s^{2}+2 \omega_{i} \zeta s+\omega_{i}^{2}},
$$


where $Q_{i}(s)$ and $B_{i}(s)$ are the Laplace transforms of $q_{i}(t)$ and $b(t)$, respectively. The inverse transformation of the transfer function will be further obtained as

$$
\begin{aligned}
& g_{i}(t) \\
& =\frac{\left(-\omega^{2}\right)\left[M U_{i N}^{(k)}(L)-r_{c} M\left(d U_{i N}^{(k)}(L) / d x\right)-m \int_{0}^{L} U_{i N}^{(k)}(x) d x\right]}{\omega_{i} \sqrt{1-\zeta^{2}}} \\
& \quad \times e^{-\omega_{i} \zeta t} \sin \left(\omega_{i} \sqrt{1-\zeta^{2}} t\right) .
\end{aligned}
$$

In this case, the solutions of (43) can be solved by convolution theorem as

$$
q_{i}(t)=b(t) * g_{i}(t)=\int_{0}^{t} b(\tau) g_{i}(t-\tau) d \tau
$$

Finally, substituting the result of (46) into (38) and after some lengthy algebraic manipulation, the absolute displacement response of the cantilever beam with tip-mass under base transverse excitation can be obtained as

$$
\begin{aligned}
u_{a}(x, \omega, t)=\sum_{i=1}^{\infty}\left\{U_{i N}^{(k)}(x) \frac{B\left[M U_{i N}^{(k)}(L)-r_{c} M\left(d U_{i N}^{(k)}(L) / d x\right)-m \int_{0}^{L} U_{i N}^{(k)}(x) d x\right]}{\sqrt{\omega^{4}+2\left(2 \zeta^{2}-1\right) \omega^{2} \omega_{i}^{2}+\omega_{i}^{4}}}\right. \\
\left.\times\left[\frac{\omega^{3}}{\omega_{i} \sqrt{1-\zeta^{2}}} e^{-\omega_{i} \zeta t} \sin \left(\omega_{i} \sqrt{1-\zeta^{2}} t+\phi_{1}\right)+\omega^{2} \sin \left(\omega t+\phi_{2}\right)\right]\right\}
\end{aligned}
$$

where $\phi_{1}$ and $\phi_{2}$ are determined by $\tan \left(\phi_{1}\right)=2 \zeta \sqrt{1-\zeta^{2}} /$ $\left(\left(\omega / \omega_{i}\right)^{2}+2 \zeta^{2}-1\right)$ and $\tan \left(\phi_{2}\right)=-2 \zeta\left(\omega / \omega_{i}\right) /\left(1-\left(\omega / \omega_{i}\right)^{2}\right)$.

It can be seen that the solution consists of two parts about time. The first term represents free vibration which will decay with time exponentially. The second term, depending on the excitation, represents the forced vibration of the system.

4.2. Experimental Responses and Comparisons with the Theoretical Results. In order to explain the absolute displacement response of the cantilever structure under base excitation experimentally, a typical cantilever beam, in which the elongated beam was inserted and fixed into the tip-mass to make sure the gravity center of tip-mass was collinear with the beam, was fabricated (as shown in Figure 9). The chosen geometric and physical properties of the cantilever beam including the tip-mass are given in Table 2.

The complete experimental devices are shown in Figure 10. The electromagnetic vibrating table type SW-TFA, controlled by a programmable controller type SW-F-2/TF-2, was used to create a specific harmonic input excitation. The cantilever beam with a tip-mass was clamped on the vibrating top platform. Eddy-Current Sensors type DM6008 and ZA210800 were used to measure the input displacement from base vibrating exciter and the absolute dynamic displacement from the beam, respectively. All measurements from cantilever beam and vibrating exciter were connected to Digital Oscilloscope type DS1204B and the Industrial Computer including a PC-based Data Acquisition Module from ADLINK company type DAQ2213. The Digital Oscilloscope and Industrial Computer displayed and processed the measurement results using the DAQPilot software and the results from subsequent analysis using the MATLAB software.
TABLE 2: Characteristic properties of the cantilever beam including tip-mass.

\begin{tabular}{lcc}
\hline Physical properties & Beam & Tip-mass \\
\hline Young's modulus, $E(\mathrm{GPa})$ & 69 & - \\
Density, $\rho_{s}$ or $\rho_{m}\left(\mathrm{~kg} / \mathrm{m}^{3}\right)$ & 2630 & 7753 \\
Length, $L$ or $L_{m}(\mathrm{~mm})$ & 120.4 & 15.6 \\
Thickness, $t_{s}$ or $t_{m}(\mathrm{~mm})$ & 4.9 & 29.5 \\
Width, $w(\mathrm{~mm})$ & 10.2 & 10.2 \\
Total mass, $M(\mathrm{~g})^{\mathrm{a}}$ & - & 33.83 \\
Offset, $r_{c}(\mathrm{~mm})^{\mathrm{b}}$ & - & 8.01 \\
Rotary inertia, $J\left(\mathrm{~kg} \cdot \mathrm{m}^{2}\right)^{\mathrm{b}, \mathrm{c}}$ & - & $3.33 \times 10^{-6}$ \\
\hline
\end{tabular}

${ }^{\mathrm{a}}$ The total mass of the tip-mass including the elongated part of beam was calculated by

$M=L_{m} t_{m} w \rho_{m}+L_{s} t_{s} w\left(\rho_{s}-\rho_{m}\right)$, where $L_{s}=10 \mathrm{~mm}$.

${ }^{\mathrm{b}}$ The offset distance and the rotary inertia were calculated according to the geometry and material properties of the beam and tip-mass using equations: $r_{c}=\left(\rho_{m} t_{m} L_{m}^{2}-\rho_{m} t_{s} L_{s}^{2}+\rho_{s} t_{s} L_{s}^{2}\right) /\left(2\left[\rho_{m} t_{m} L_{m}+L_{s} t_{s}\left(\rho_{s}-\rho_{m}\right)\right]\right)$, $J=\rho_{m} L_{m} t_{m} w\left[(1 / 12)\left(L_{m}^{2}+t_{m}^{2}\right)+\left((1 / 2) L_{m}-r_{c}\right)^{2}\right]+L_{s} t_{s} w\left(\rho_{s}-\right.$ $\left.\rho_{m}\right)\left[(1 / 12)\left(L_{s}^{2}+t_{s}^{2}\right)+\left(r_{c}-(1 / 2) L_{s}\right)^{2}\right]$.

${ }^{c}$ The corresponding parameters $\phi, k / L$, and $r_{c} / L$ were calculated as follows: $\phi=M /\left(\rho_{s} t_{s} w\right)=2.1377, k / L=\sqrt{J / M} / L=0.0824$, and $r_{c} / L=0.0665$.

The analytical model defined by (47), in which two types of mode shapes derived by two improved analytical methods are utilized, can be used to analyze the steady and transient displacement responses of the cantilever beam with a tipmass and an input base excitation based on the chosen varying frequencies, time, and positions along the length of the beam. Typically, the displacement responses from positions along $x$-axis at $x_{1}=36.4 \mathrm{~mm}, x_{2}=57.4 \mathrm{~mm}$, $x_{3}=78.4 \mathrm{~mm}, x_{4}=99.4 \mathrm{~mm}$ and $x_{5}=120.4 \mathrm{~mm}$ off the origin point (as shown in Figure 9) were measured 


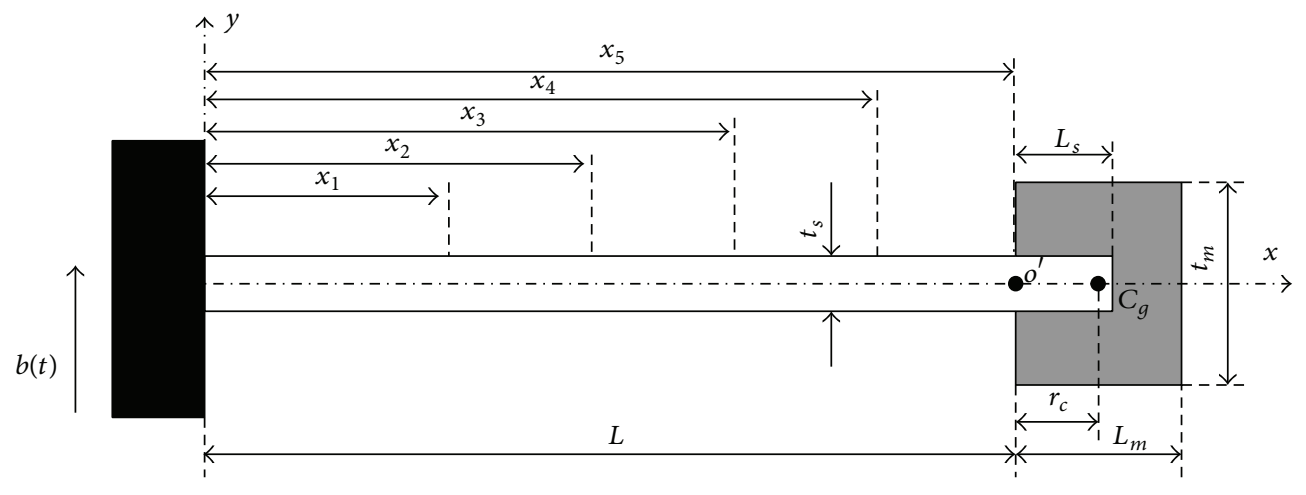

FIGURE 9: Typical cantilever beam with a tip-mass.
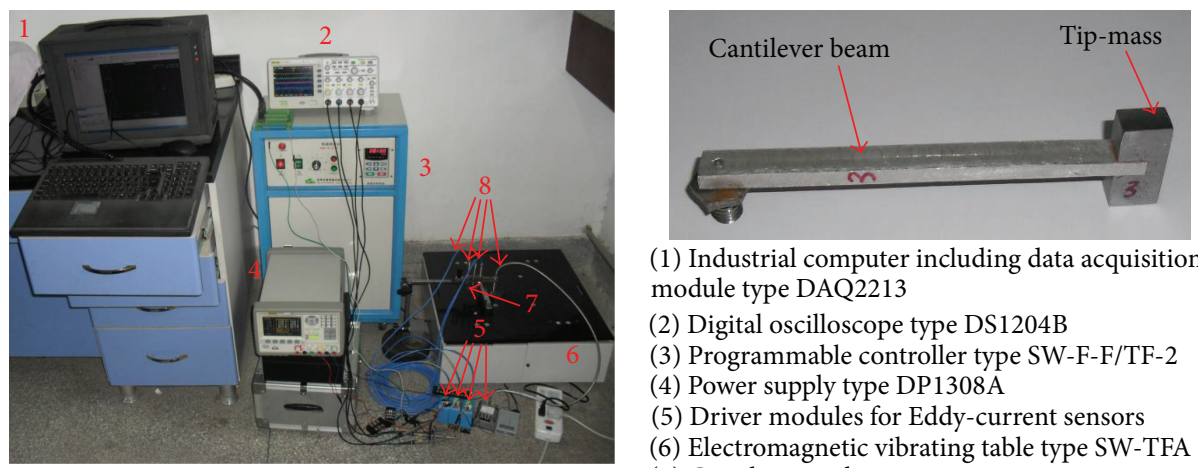

(1) Industrial computer including data acquisition module type DAQ2213

(2) Digital oscilloscope type DS1204B

(3) Programmable controller type SW-F-F/TF-2

(4) Power supply type DP1308A

(5) Driver modules for Eddy-current sensors

(6) Electromagnetic vibrating table type SW-TFA

(7) Cantilever with a tip-mass

(8) Eddy-current sensors type DM6008 and ZA210800

FIGURE 10: Complete experimental setup.

by Eddy-Current Sensors. The amplitude of the input sinedisplacement excitation for analysis was measured as $B=$ $0.01 \mathrm{~mm}$ and the damping ratio for the transverse vibration around the fundamental resonant frequency was found to be $\zeta=0.0025$ by Logarithmic Decrement Method.

In the following paragraphs, we focus on the transient and steady responses of the cantilever system with a sine base excitation.

Figures 11 and 12 show the theoretical displacement responses at the free end of the beam from transient to steady state with two improved mode shapes above at the first resonant frequency. It can be seen obviously that the displacement responses with two improved mode shapes we proposed are almost identical. This richly proves that the first-type mode shapes reformulated by the reinforced method of separation of variables are equal to the secondtype mode shapes derived by the developed method of forced vibration. In addition, it can be further obtained that, when the frequency from the base excitation is very close but not exactly equal to the resonant frequency of free vibrations of the cantilever system, a kind of vibration called beating occurs and the period of the beating increases as $\omega$ approached $\omega_{1}$. This kind of beating decays exponentially along the time due to the mechanical damping.

Figure 13 shows the experimental displacement responses at the position of $x=120.4 \mathrm{~mm}$ from transient to steady state. As the same to the theoretical results, experimental displacement response can be regarded as the sum of waveforms from transient and steady states. But the transient response vanished gradually with the time due to the mechanical damping and only the steady response left. In addition, the phenomenon of beating still existed. The amplitude of this beating phenomenon reduced gradually and the closer the exciting frequency approached to the first natural frequency of the cantilever system, the faster the amplitude of the beating decreased.

Furthermore, the amplitudes of the theoretical waveforms in Figures 11 and 12 are different from the experimental result in Figure 13. This may because af the following. (a) The effect of mechanical damping with respect to stiffness was only considered and the mass damping was ignored in theoretical analysis which can also weaken the transient vibration faster. (b) The first natural frequency of the cantilever system was calculated and measured by theoretical and experimental methods as $81.5 \mathrm{~Hz}$ and $81.2 \mathrm{~Hz}$, respectively. The little difference made that the max amplitudes from theoretical and experimental results did not appear at the same frequency. (c) In theoretical analysis, the tip-mass was equated by the inertia force and inertia moment acting at the free end of the cantilever, so the total length for theoretical analysis was just the length of the beam of $120.4 \mathrm{~mm}$. However, during experiments, the tip-displacement was measured at 

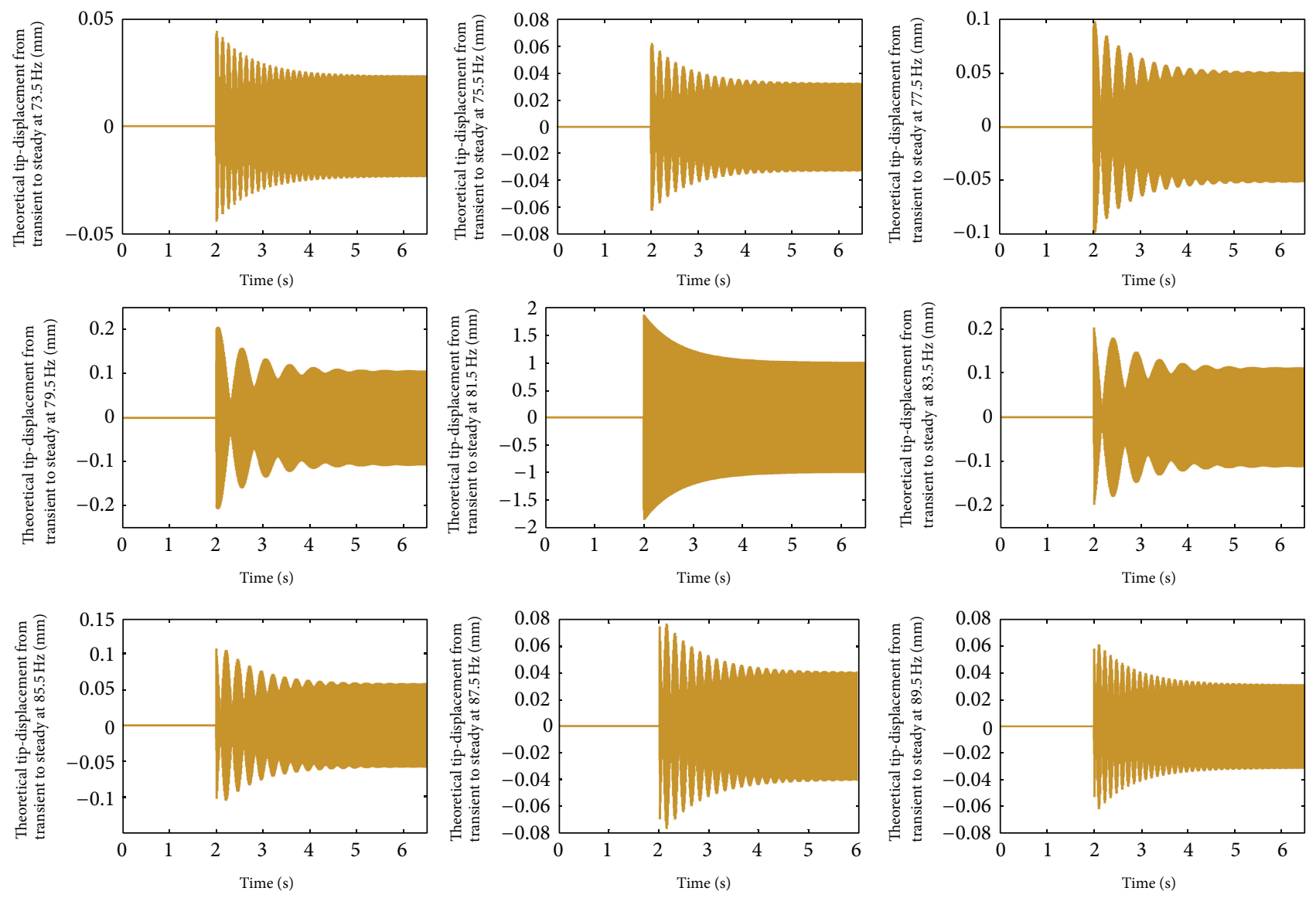

FIGURE 11: Theoretical tip displacement responses with the second-type mode shape of the beam from transient to steady state.

the approximate position of $x=120.4 \mathrm{~mm}$. This is just an approximate value because Eddy-Current Sensor cannot give exact measurement results of the displacement at the junction between the cantilever and the tip-mass. This may cause that the amplitude level from theoretical discussion was higher than from experimental measurement. In addition, the amplitude of the instantaneous base displacement from the exciter may be not the fixed value $0.01 \mathrm{~mm}$ at the starting instant. Therefore, the maximum displacement responses during the transient state in the experimental measurements may shift away from the real experimental results.

The theoretical responses with two improved types of mode shapes and the experimental displacement responses versus varying positions of beam and the frequencies at steady state are shown in Figures 14-16, respectively. Figures 14 and 15 show two types of theoretical displacements at the positions of $x=36.4 \mathrm{~mm}, 57.4 \mathrm{~mm}, 78.4 \mathrm{~mm}, 99.4 \mathrm{~mm}$, and $120.4 \mathrm{~mm}$ with continuous varying frequencies. In experiments, the displacement responses were measured at the same positions with theoretical calculations but with some certain frequencies of $78.2 \mathrm{~Hz}, 79.2 \mathrm{~Hz}, 80.2 \mathrm{~Hz}, 80.7 \mathrm{~Hz}$, $81 \mathrm{~Hz}, 81.2 \mathrm{~Hz}, 81.7 \mathrm{~Hz}, 82 \mathrm{~Hz}, 83 \mathrm{~Hz}$, and $84 \mathrm{~Hz}$ as plotted in Figure 16. It is noticed that when the frequency of base excitation approached to the first natural frequency of the cantilever system, the amplitude of forced vibration will rapidly increase and become maximum for the case when the frequency of the excitation coincides with the natural frequency. Table 3 lists the values of the first natural frequencies and the displacement responses from theoretical and experimental results at some certain positions long the beam under the first natural frequencies. It can be seen that the theoretical and experimental results are matched very close.

Table 3: The first natural frequencies and the resonant displacements at some certain positions.

In a word, the theoretical results with two different mode shapes derived by two improved analytical methods have extremely the same behaviors at transient and steady states with respect to varying frequencies from base excitation. The displacement responses indicate close agreement between the experimental and analytical results. All of these results give full verification for the improvement of the analytical methods in this paper utilized in recalculating the mode shapes of a cantilever beam when an offset distance exists between the gravity center of the tip-mass and the point of attachment.

\section{Conclusions}

This paper presents two improved analytical methods of calculation for natural frequencies and mode shapes of a uniform cantilever beam carrying a tip-mass under an input 

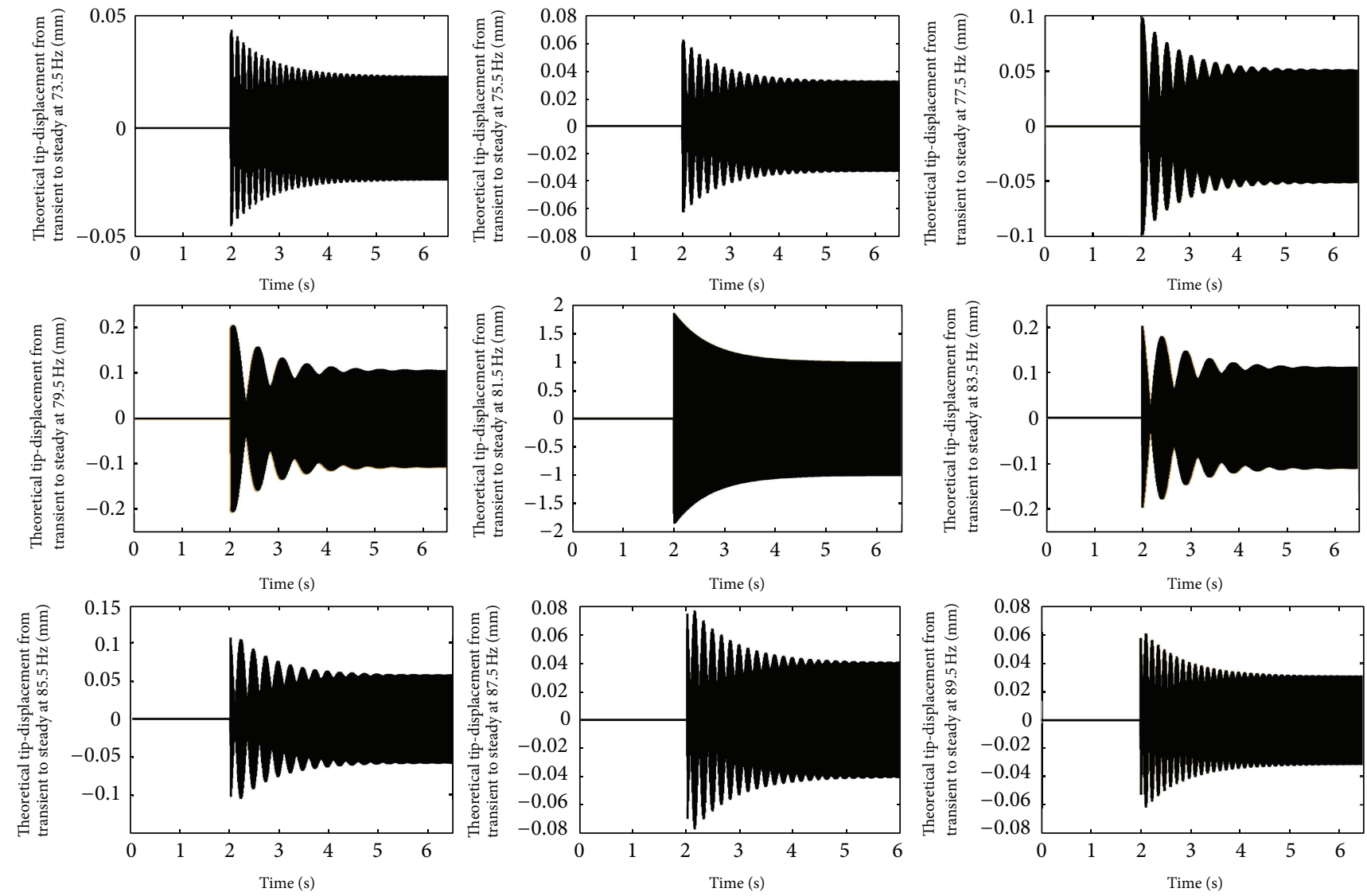

FIGURE 12: Theoretical tip displacement responses with third-type mode shape of the beam from transient to steady state.

TABle 3: The first natural frequencies and the resonant displacements at some certain positions.

\begin{tabular}{|c|c|c|c|c|}
\hline \multirow{2}{*}{ Resonant frequency or positions } & \multicolumn{3}{|c|}{ The values of frequencies and displacement responses ( $\mathrm{Hz}$ or $\mathrm{mm})$} & \multirow{2}{*}{ Errors } \\
\hline & Theory based on $U_{1}^{(2)}(x)$ & Theory based on $U_{1}^{(3)}(x)$ & Experiment & \\
\hline The 1st natural frequency & 81.5 & 81.5 & 81.2 & $0.37 \%$ \\
\hline$x=36.4 \mathrm{~mm}$ & 0.1285 & 0.1285 & 0.1277 & $0.62 \%$ \\
\hline$x=57.4 \mathrm{~mm}$ & 0.3022 & 0.3022 & 0.3001 & $0.69 \%$ \\
\hline$x=78.4 \mathrm{~mm}$ & 0.5279 & 0.5279 & 0.5246 & $0.63 \%$ \\
\hline$x=99.4 \mathrm{~mm}$ & 0.7905 & 0.7905 & 0.7855 & $0.63 \%$ \\
\hline$x=120.4 \mathrm{~mm}$ & 1.0742 & 1.0742 & 1.0671 & $0.66 \%$ \\
\hline
\end{tabular}

base excitation using forced vibration theory and the method of separation of variables. A simplified cantilever model by replacing the tip-mass with an equivalent inertial force and inertial moment acting at the free end of the cantilever beam was detailed. In addition, using the Dirac Delta Function, the base transverse excitation, the concentrated equivalent inertia force, and inertia moment were represented in the form of distributed loads, which can make the method of separation of variables be utilized properly. In this case, some typical results of natural frequencies and mode shapes of the cantilever beam with a tip-mass were derived using the reinforced method of separation of variables and the developed forced vibration, respectively. Furthermore, based on modal analysis method, the transient and steady displacement responses of the cantilever system with a tip-mass and a sine base excitation were presented in which two different types of mode shapes calculated by two improved analytical methods were used. Finally, an experimental cantilever system was fabricated and some practical displacement responses were measured.

From the results of the typical natural frequencies and mode shapes, we can easily found that the mode shapes from the first improved type were identical to the mode shapes from the second type whether the mode shapes from the lower order or from the higher order even the offset distance was large. The comparing results of the transient and steady displacement responses from the theoretical analysis and experimental measurements further proved the 

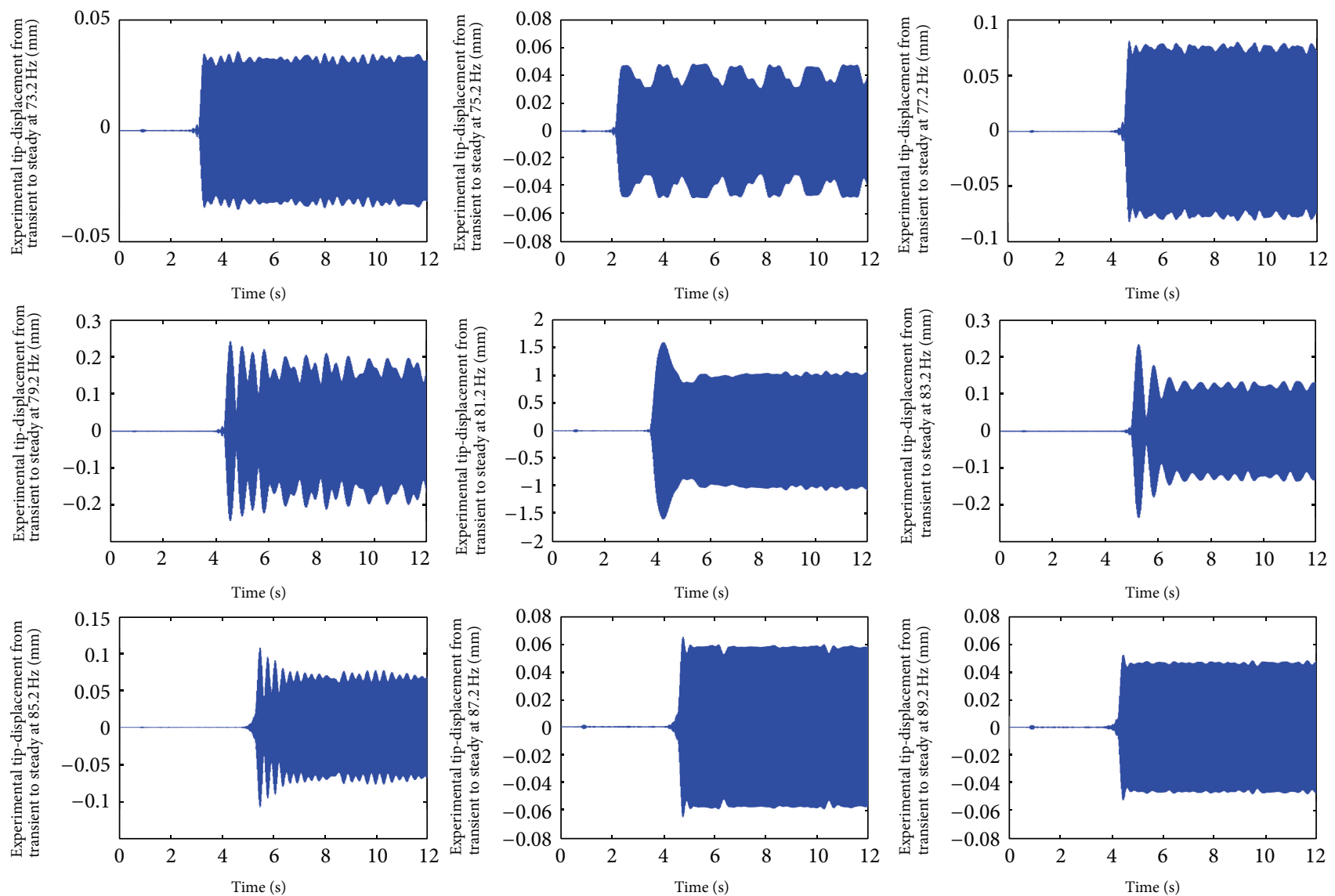

FIGURE 13: Experimental displacement responses at the position of $x=120.4 \mathrm{~mm}$ from transient to steady state.

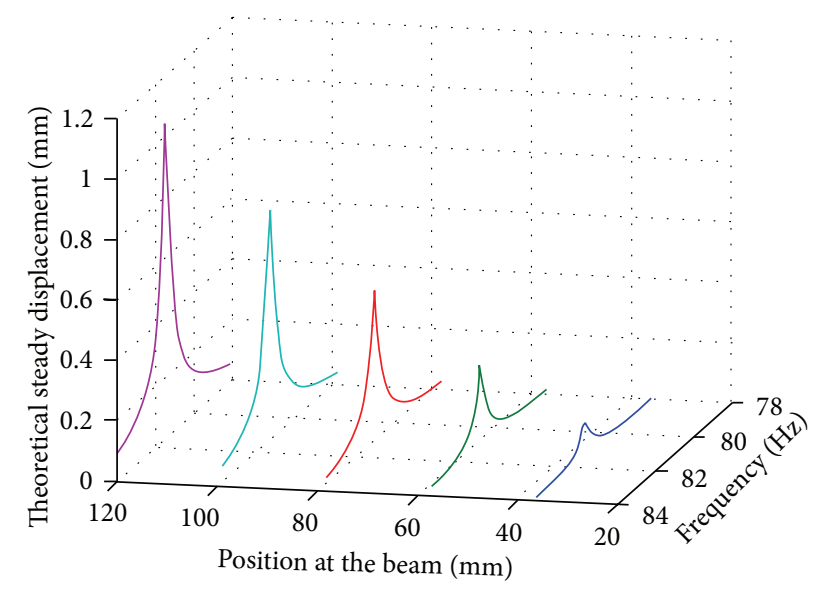

Figure 14: Theoretical steady-displacement responses versus positions and frequencies with mode $U_{1}^{(2)}(x)$.

improvements of the analytical methods for calculating the mode shapes of the cantilever beam with a tip-mass and a base excitation. In addition, the amplitudes of the mode shapes decreased with the increasing mass value of the tipmass when the moment of inertia and offset were constants. The amplitude of the first-order mode shape increased, but

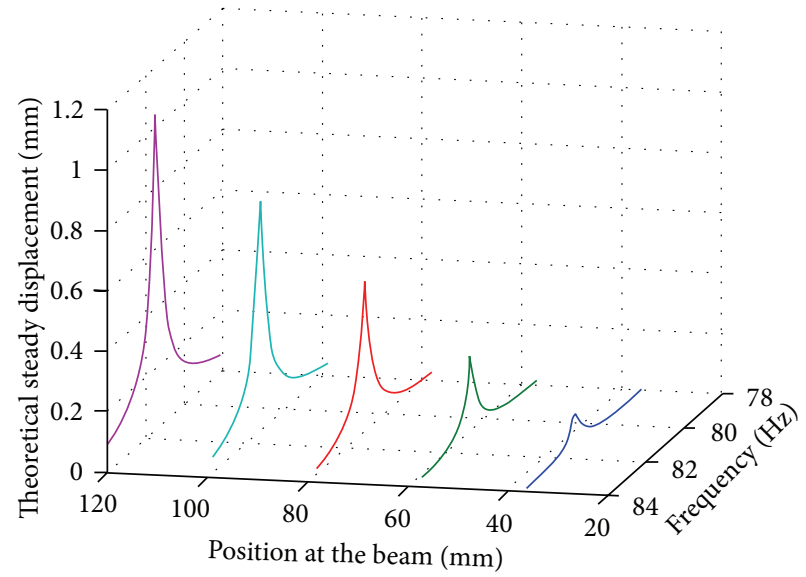

FIGURE 15: Theoretical steady-displacement responses versus positions and frequencies with mode $U_{1}^{(3)}(x)$.

the other nine mode shapes decreased with the increasing moment of inertia when the mass and offset were set as constants. Conversely, the amplitude of the first mode shape decreased, but the other nine mode shapes increased with the increasing offset distance under the condition that the mass and moment of inertia were constants. Globally, the effect 


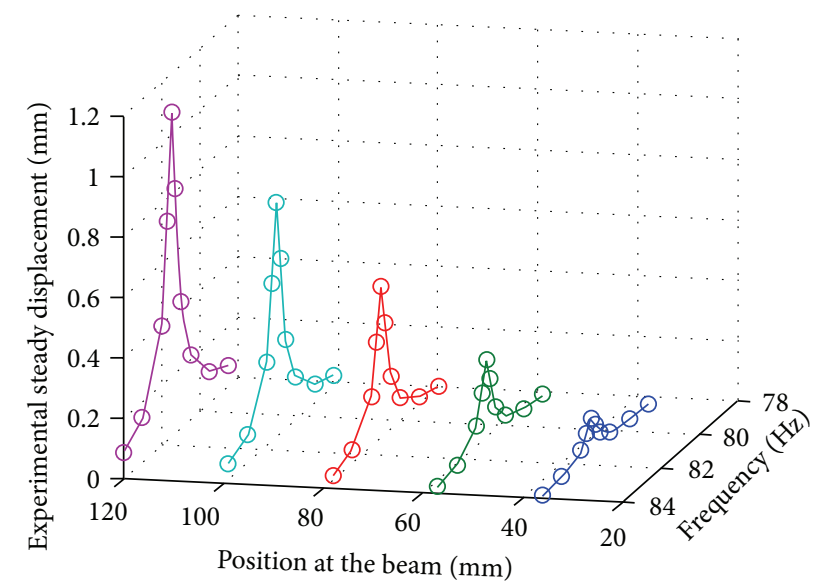

FIGURE 16: Experimental steady-displacement responses versus positions and frequencies.

from varying combinations of the mass and moment inertia of the tip-mass and the offset distance for the lower-order mode shapes was stronger than for the higher ones.

Furthermore, it is clearly noticed that the mass of tipmass can reduce the values of the natural frequencies and the increasing mass value gave the decreasing frequencies when the moment of inertia of the tip-mass and the offset distance were set as constants. Similarly, the moment of inertia can further decrease the natural frequencies and the higher moment of inertia resulted in the lower frequencies when the mass and the offset were constants. Differently, the offset distance can reduce the first-order natural frequencies but increase the other nine order frequencies, and the higher offset distance led to the lower first-order natural frequencies but produced the higher other nine order frequencies when the mass and the moment of inertia were set to constant. In global view, the influence from the varying mass, moment of inertia, and offset distance for the lower-order natural frequencies was larger than for the higher-order ones weather this influence was negative or positive.

\section{Conflict of Interests}

The authors declare that there is no conflict of interests regarding the publication of this paper.

\section{Acknowledgments}

This work is supported by the Natural Science Foundations of China (no. 51275380) and the Scientific and Technological Project of Shaanxi Province (no. 2012K06-36). The authors would like to thank the reviewers for their valuable comments on this paper.

\section{References}

[1] P. A. A. Laura, J. L. Pombo, and E. A. Susemihl, "A note on the vibrations of a clamped-free beam with a mass at the free end," Journal of Sound and Vibration, vol. 37, no. 2, pp. 161-168, 1974.
[2] B. Rama Bhat and H. Wagner, "Natural frequencies of a uniform cantilever with a tip mass slender in the axial direction," Journal of Sound and Vibration, vol. 45, no. 2, pp. 304-307, 1976.

[3] C. W. S. To, "Vibration of a cantilever beam with a base excitation and tip mass," Journal of Sound and Vibration, vol. 83, no. 4, pp. 445-460, 1982.

[4] P. A. A. Laura, "Comments on 'Vibration of a cantilever beam with a base excitation and tip mass"' Journal of Sound and Vibration, vol. 88, no. 4, p. 569, 1983.

[5] C. W. S. To, "Discussion on 'vibration of a cantilever beam with a base excitation and tip mass"' Journal of Sound and Vibration, vol. 97, no. 2, pp. 349-351, 1984.

[6] R. G. Jacquot, "Further comments on 'Vibration of a cantilever beam with a base excitation and tip mass,' Journal of Sound and Vibration, vol. 93, no. 2, pp. 312-313, 1984.

[7] S. A. Mousavi Lajimi and G. R. Heppler, "Comments on 'natural frequencies of a uniform cantilever with a tip mass slender in the axial direction,' Journal of Sound and Vibration, vol. 331, no. 12, pp. 2964-2968, 2012.

[8] R. B. Bhat, "Authors response to the comments on 'natural frequencies of a uniform cantilever with a tip mass slender in the axial direction," Journal of Sound and Vibration, vol. 331, no. 12, pp. 2962-2963, 2012.

[9] E. Esmailzadeh and G. Nakhaie-Jazar, "Periodic behavior of a cantilever beam with end mass subjected to harmonic base excitation," International Journal of Non-Linear Mechanics, vol. 33, no. 4, pp. 567-577, 1998.

[10] H. Abramovich and O. Hamburger, "Vibration of a cantilever timoshenko beam with a tip mass," Journal of Sound and Vibration, vol. 148, no. 1, pp. 162-170, 1991.

[11] B. Pratiher, "Vibration control of a transversely excited cantilever beam with tip mass," Archive of Applied Mechanics, vol. 82, no. 1, pp. 31-42, 2012.

[12] M. Kim, M. Hoegen, J. Dugundji, and B. L. Wardle, "Modeling and experimental verification of proof mass effects on vibration energy harvester performance," Smart Materials and Structures, vol. 19, Article ID 045023, 2010.

[13] Y. T. Li and R. Wong, "Integral and series representations of the Dirac delta function," Communications on Pure and Applied Analysis, vol. 7, no. 2, pp. 229-247, 2008.

[14] M. Al-Gwaiz, Sturm-Liouville Theory and its Applications, chapter 3, Springer, London, UK, 2008.

[15] F. Bao-lian, "Generalized reciprocal theorems and their applications," Applied Mathematics and Mechanics, vol. 23, no. 2, pp. 203-210, 2001.

[16] C. Cai, H. Zheng, M. Khan, and K. Hung, Modeling of Material Damping Properties in ANSYS, Defense Systems Division, Institute of High Performance Computing, 2002. 

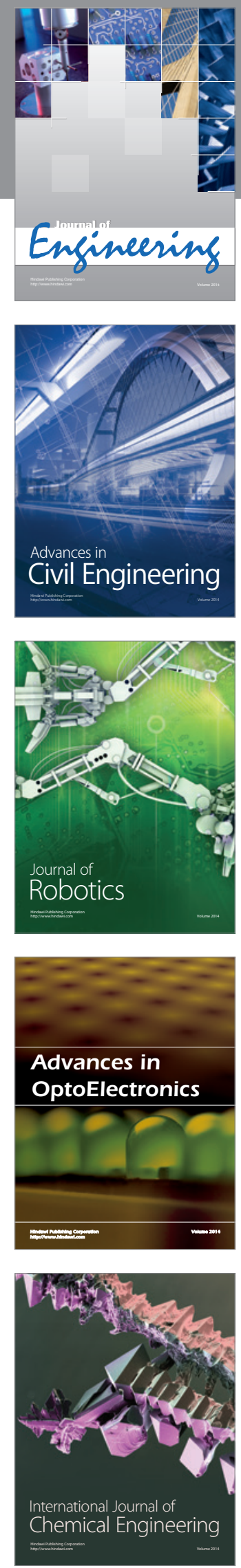

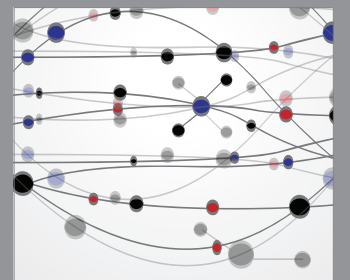

The Scientific World Journal
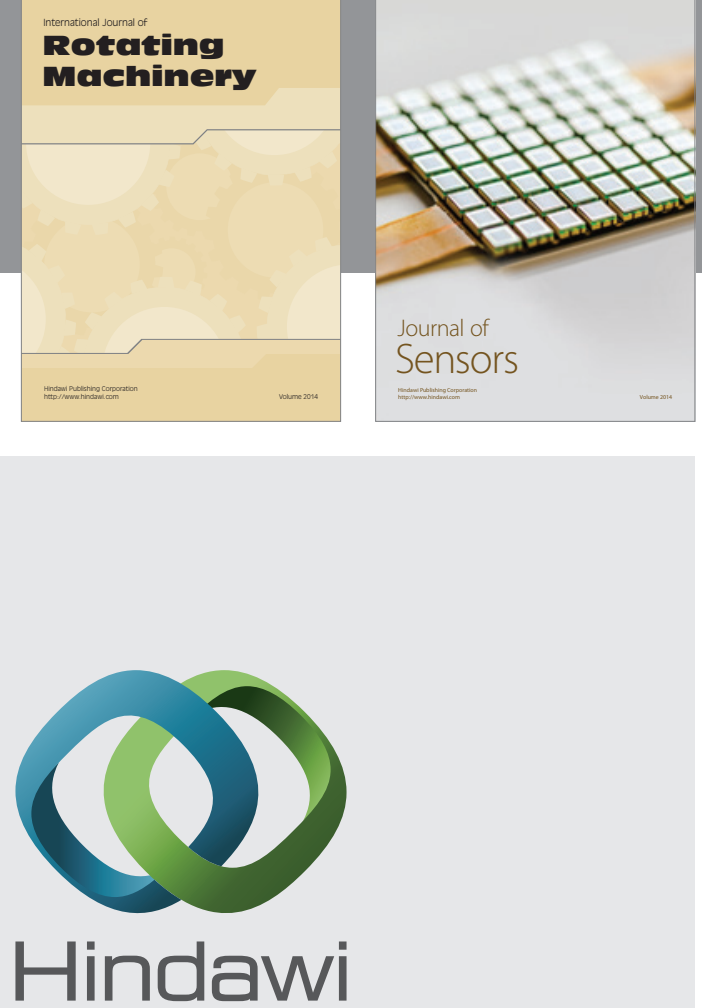

Submit your manuscripts at http://www.hindawi.com
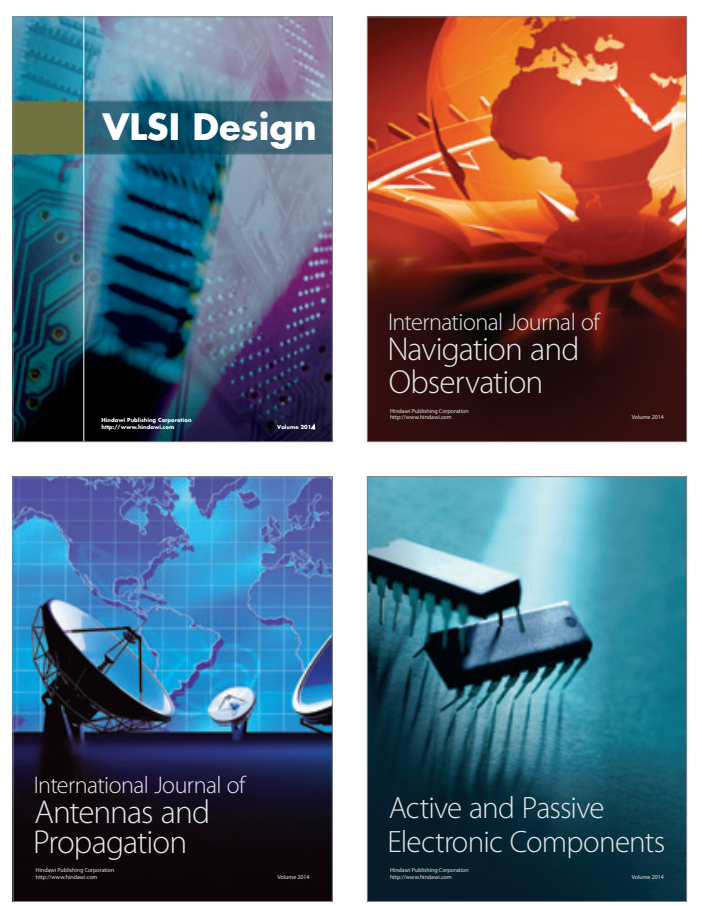
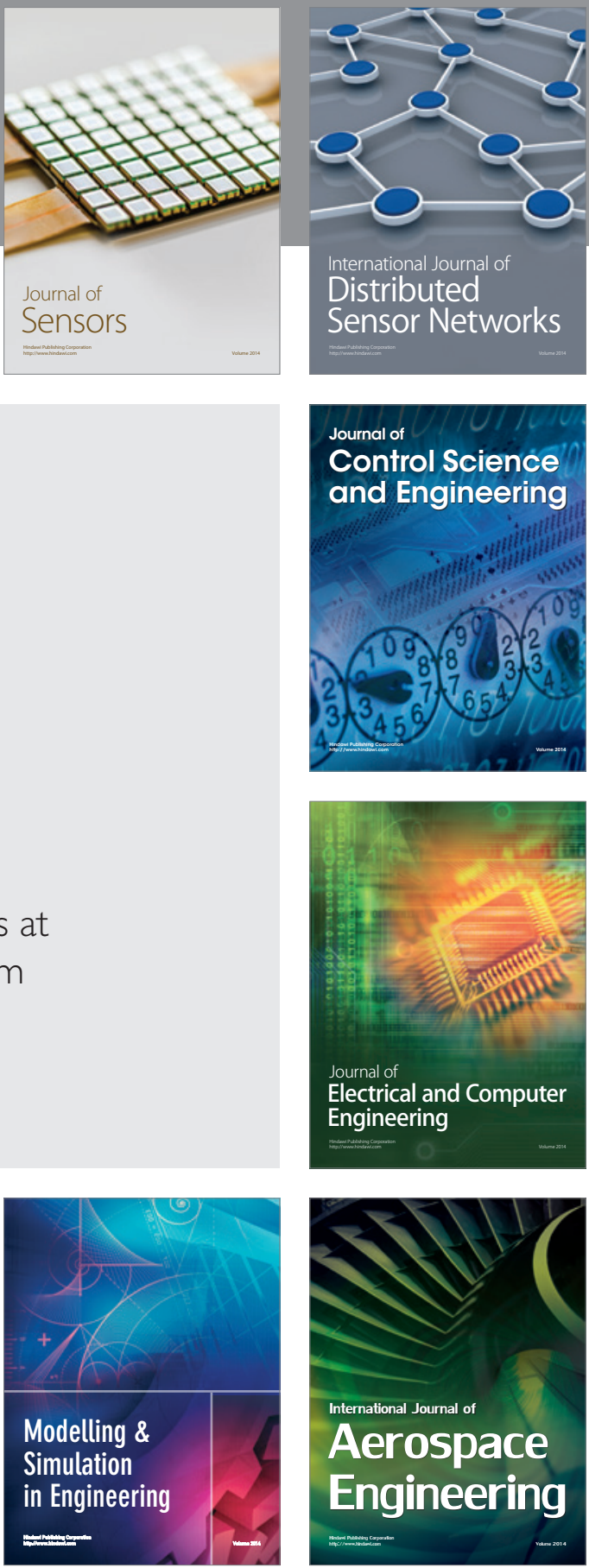

Journal of

Control Science

and Engineering
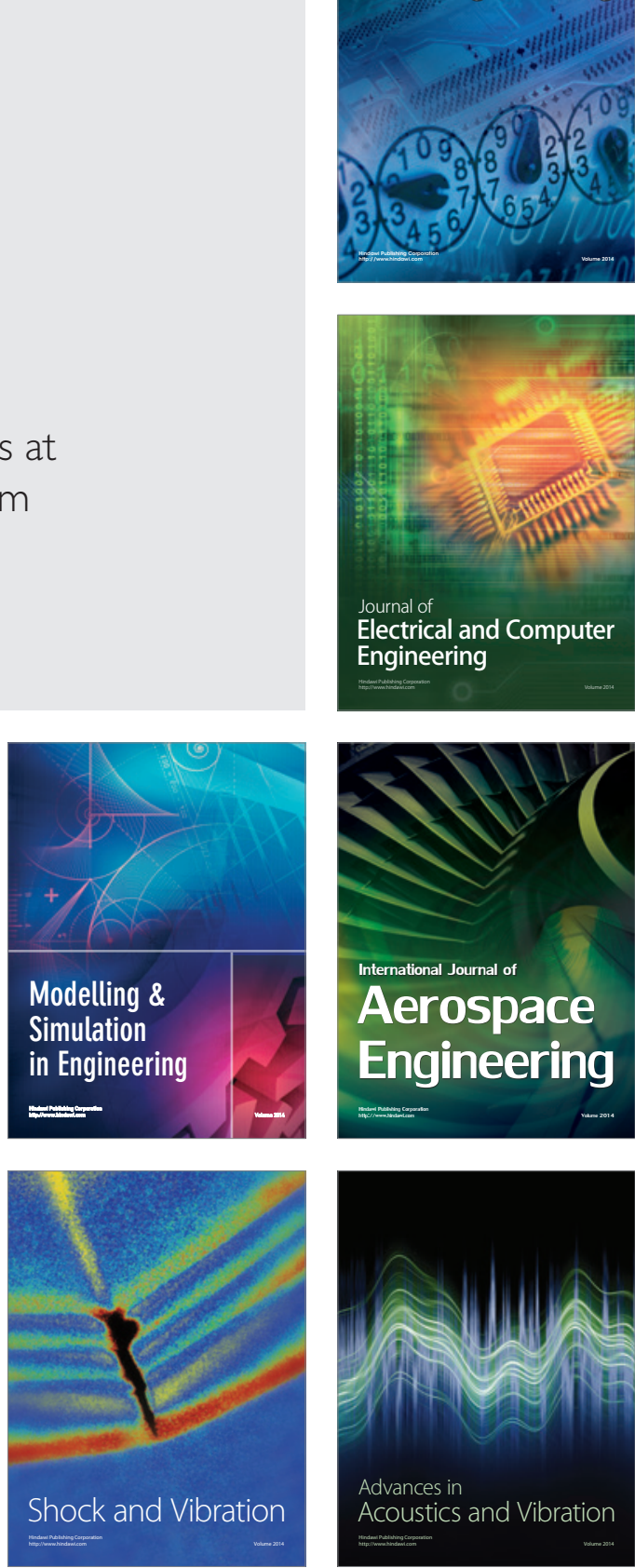